\title{
NON-ELEMENTARY COMPLEXITIES FOR BRANCHING VASS, MELL, AND EXTENSIONS
}

\author{
RANKO LAZIĆ AND SYLVAIN SCHMITZ
}

\begin{abstract}
We study the complexity of reachability problems on branching extensions of vector addition systems, which allows us to derive new non-elementary complexity bounds for fragments and variants of propositional linear logic. We show that provability in the multiplicative exponential fragment is TowER-hard already in the affine case - and hence non-elementary. We match this lower bound for the full propositional affine linear logic, proving its TOWER-completeness. We also show that provability in propositional contractive linear logic is ACKERMANN-complete.

KeY Words. Linear logic, vector addition systems, fast-growing complexity.
\end{abstract}

\section{INTRODUCTION}

The use of various classes of counter machines to provide computational counterparts to propositional substructural logics has been highly fruitful, allowing to prove for instance:

- the undecidability of provability in propositional linear logic (LL), thanks to a reduction from the halting problem in Minsky machines proved by Lincoln, Mitchell, Scedrov, and Shankar [18, who initiated much of this line of work,

- the decidability of the !-Horn fragment of multiplicative exponential linear logic, proved by Kanovich [13] by reduction to reachability in vector addition systems,

- the decidability of provability in affine linear logic, first shown by Kopylov using a notion of vector addition games [14,

- the ACKERMANN-completeness of provability in the conjunctive implicative fragment of relevance logic, proved by Urquhart [30], using reductions to and from expansive alternating vector addition systems, and

- the inter-reducibility between provability in multiplicative exponential linear logic and reachability in a model of branching vector addition systems, shown by de Groote, Guillaume, and Salvati [9].

1.1. Alternating Branching VASS. In this paper, we revisit the correspondences between propositional linear logic and counter systems with a focus on computational complexity. In Section 3, we define a model of alternating branching vector addition systems (ABVASS) with full zero tests. While this model can be seen as an extension and repackaging of Kopylov s vector games, its reachability problem enjoys very simple reductions to and

Work partially supported by ANR grant ReacHard 11-BS02-001-01. 
from provability in LL, which are suitable for complexity statements (see Section 4). We prove that:

- coverability in the top-down, root-to-leaves direction is TOwERcomplete, i.e. complete for the class of problems that can be solved with time or space resources bounded by a tower of exponentials whose height depends elementarily on the input size (see Section 5 for the upper bound and Section 6 for the lower bound), and

- coverability in the bottom-up, leaves-to-root direction and so-called "meet" and "zero-jump" semantics is complete for ACKERMANN, i.e. complete for resources bounded by the Ackermann function of some primitive-recursive function of the input (see Section 7).

1.2. Provability in Substructural Logics. Our complexity bounds for ABVASS translate into the exact same bounds for provability in fragments and variants of LL:

1.2.1. Affine Linear Logic (LLW) was proved decidable by Kopylov [14] in 1995 using vector addition games; a model-theoretic proof was later presented by Lafont [15].1]

The best known complexity bounds for LLW are due to Urquhart [31: by a reduction from coverability in vector addition systems [19], he derives an ExpSPACE lower bound, very far from the ACKERMAnN upper bound he obtains from length function theorems for Dickson's Lemma [see e.g. 12].

1.2.2. Contractive Linear Logic (LLC) was proved decidable by Okada and Terui [22] by model-theoretic methods.

Urquhart [30] showed the ACKERMANN-completeness of provability in a fragment of relevance logic, which is also a fragment of intuitionistic multiplicative additive LLC. To the best of our knowledge, there are no known complexity upper bounds for provability in LLC.

1.2.3. Multiplicative Exponential Linear Logic. The main open question in this area is whether the multiplicative exponential fragment (MELL) is decidable. It is related to many decision problems, for instance in computational linguistics [24, 25], cryptographic protocol verification [32], the verification of parallel programs [4, and data logics [3, 11].

Thanks to the reductions to and from the reachability problem in branching vector addition systems with states (BVASS) [9] and to the bounds of Lazić [17], we know that provability in MELL is 2-EXPSPACE-hard.

\subsubsection{Summary of the Complexity Results:}

LLW: We improve both the lower bound and the upper bound of Urquhart [31], and prove that LLW provability is complete for TowER.

\footnotetext{
${ }^{1}$ Variants of LLW are popular in the literature on implicit complexity; for instance, light affine linear logic [2] is known to type exactly the class FP of polynomial time computable functions. In this paper we are however interested in the complexity of the provability problem (for the propositional fragment), rather than the complexity of normalisation. In terms of typed lambda calculi, our results pertain to the complexity of the type inhabitation problem.
} 
LLC: We show that LLC provability is ACKERMAnN-complete; the lower bound already holds for the multiplicative additive fragment MALLC.

MELL: Our TOwER-hardness result for LLW already holds for affine MELL and thus for MELL, which improves over the 2-ExPSPACE lower bound of Lazić [17].

ILL: All of our complexity bounds also hold for provability in the intuitionistic versions of our calculi. See $\$ 4.1 .2$ for details.

\section{Propositional Linear Logic}

2.1. Classical Linear Logic. For convenience, we present here a sequent calculus for classical propositional linear logic that works with formulæ in negation normal form and considers one-sided sequents [see e.g. 29].

2.1.1. Syntax. Propositional linear logic formulæ are defined by the abstract syntax

$$
\begin{aligned}
A, B::= & a \mid a^{\perp} \\
& \mid A \text { \& } B|A \otimes B| \perp \mid \mathbf{1} \\
& |A \& B| A \oplus B|\top| \mathbf{0} \\
& |! A| ? A
\end{aligned}
$$

where $a$ ranges over atomic formulæ. We write " $A^{\perp}$ " for the negation normal form of $A$, where negations are pushed to the atoms using the dualities $A^{\perp \perp}=A,(A \ngtr B)^{\perp}=A^{\perp} \otimes B^{\perp}, \perp^{\perp}=\mathbf{1},(A \& B)^{\perp}=A^{\perp} \oplus B^{\perp}, \top^{\perp}=\mathbf{0}$, and $(? A)^{\perp}=! A^{\perp}$. We write " $A \multimap B$ " for the linear implication $A^{\perp}$ >8 $B$.

2.1.2. Sequent Calculus. The rules of the sequent calculus manipulate multisets of formulæ, denoted by $\Gamma, \Delta, \ldots$, so that the exchange rule is implicit; "? $\Gamma$ " then denotes a multiset of formulæ all guarded by why-nots: ? $\Gamma$ is of the form $? A_{1}, \ldots, ? A_{n}$.

$$
\begin{aligned}
& \frac{\vdash \Gamma, A^{\perp}}{\vdash n i t} \quad \frac{\vdash \Gamma, A \quad \vdash \Delta, A^{\perp}}{\vdash \Gamma, \Delta} \text { cut } \\
& \frac{\vdash \Gamma, A, B}{\vdash \Gamma, A \ngtr B} \ngtr \quad \frac{\vdash \Gamma, A \quad \vdash \Delta, B}{\vdash \Gamma, \Delta, A \otimes B} \otimes \frac{\vdash \Gamma}{\vdash \Gamma, \perp} \perp \quad \overline{\vdash \mathbf{1}} \mathbf{1} \\
& \frac{\vdash \Gamma, A \quad \vdash \Gamma, B}{\vdash \Gamma, A \& B} \& \quad \frac{\vdash \Gamma, A}{\vdash \Gamma, A \oplus B} \frac{\vdash \Gamma, B}{\vdash \Gamma, A \oplus B} \oplus \overline{\vdash \Gamma, \top}^{\top} \\
& \frac{\vdash \Gamma, A}{\vdash \Gamma, ? A} \text { ?D } \quad \frac{\vdash \Gamma}{\vdash \Gamma, ? A} \text { ?W } \quad \frac{\vdash \Gamma, ? A, ? A}{\vdash \Gamma, ? A} \text { ?C } \quad \frac{\vdash ? \Gamma, A}{\vdash ? \Gamma, ! A} \text { ?P }
\end{aligned}
$$

The last four rules for exponential formulæ are called dereliction (?D), logical weakening (?W), logical contraction (?C), and promotion (?P).

The cut rule can be eliminated in this calculus, which then enjoys the subformula property: in any rule except cut, the formulæ appearing in the premises are subformulæ of the formulæ appearing in the conclusion. 
2.2. Fragments and Variants. Lincoln et al. [18] established most of the results on the decidability and complexity of provability in propositional linear logic. In particular, the full propositional linear logic (LL) is undecidable, while its multiplicative additive fragment (MALL, which excludes the exponential connectives and rules) is decidable in polynomial space. As mentioned in the introduction, the main open question in this area is whether the multiplicative exponential fragment (MELL, which excludes the additive connectives and rules) is decidable.

Regarding related logics, the structural rules of structural weakening (W) and structural contraction $(\mathrm{C})$

$$
\frac{\vdash \Gamma}{\vdash \Gamma, A} \mathrm{~W} \quad \frac{\vdash \Gamma, A, A}{\vdash \Gamma, A} \mathrm{C}
$$

give rise to two decidable variants of LL. If we replace logical weakening (?W) by structural weakening (W), we define affine linear logic (LLW). If we similarly replace logical contraction (?C) by structural contraction (C), we define contractive linear logic (LLC). The sequent calculi for LLW and LLC also enjoy cut elimination and the subformula property for cut-free proofs.

2.2.1. Intuitionistic Linear Logic. Intuitionistic linear logic is essentially obtained from classical linear logic by restricting its two-sided sequent calculus to consequents (the right sides of sequents) with at most one formula. We present here a variant of intuitionistic linear logic with bottom [29, Section 2.5], which we will refer to as ILZ:

$$
\begin{aligned}
& \overline{A \vdash A} \text { init } \quad \frac{\Gamma \vdash A \quad \Delta, A \vdash B}{\Gamma, \Delta \vdash B} \text { cut } \\
& \frac{\Gamma \vdash A \quad \Delta, B \vdash C}{\Gamma, \Delta, A \multimap B \vdash C} \mathrm{~L}_{\frown} \quad \frac{\Gamma, A \vdash B}{\Gamma \vdash A \multimap B} \mathrm{R}_{\odot} \\
& \frac{\Gamma, A, B \vdash C}{\Gamma, A \otimes B \vdash C} \mathrm{~L}_{\otimes} \quad \frac{\Gamma \vdash A \quad \Delta \vdash B}{\Gamma, \Delta \vdash A \otimes B} \mathrm{R}_{\otimes} \\
& \frac{\Gamma \vdash}{\perp \vdash} \mathrm{L}_{\perp} \quad \frac{\Gamma \vdash \perp}{\Gamma \vdash} \\
& \frac{\Gamma \vdash A}{\Gamma, \mathbf{1} \vdash A} \mathrm{~L}_{1} \quad \frac{\vdash \mathbf{1}}{\mathrm{R}_{1}} \\
& \frac{\Gamma, A \vdash C \quad \Gamma, B \vdash C}{\Gamma, A \oplus B \vdash C} \mathrm{~L}_{\oplus} \quad \frac{\Gamma \vdash A}{\Gamma \vdash A \oplus B} \frac{\Gamma \vdash B}{\Gamma \vdash A \oplus B} \mathrm{R}_{\oplus} \\
& \frac{\Gamma, A \vdash C}{\Gamma, A \& B \vdash C} \frac{\Gamma, B \vdash C}{\Gamma, A \& B \vdash C} \mathrm{~L}_{\&} \quad \frac{\Gamma \vdash A \quad \Gamma \vdash B}{\Gamma \vdash A \& B} \mathrm{R}_{\&} \\
& \overline{\Gamma \vdash \mathrm{T}} \mathrm{R}_{\mathrm{T}} \\
& \frac{\Gamma, A \vdash B}{\Gamma, ! A \vdash B} \text { !D } \quad \frac{\Gamma \vdash B}{\Gamma, ! A \vdash B} \text { !W } \quad \frac{\Gamma, ! A, ! A \vdash B}{\Gamma, ! A \vdash B} \text { !C } \quad \frac{! \Gamma \vdash A}{! \Gamma \vdash ! A} \text { !P }
\end{aligned}
$$

The fragment without $\perp$ is better known as ILL. 
2.2.2. Affine and Contractive Variants. The intuitionistic versions with bottom ILZW and ILZC and without bottom ILLW and ILLC of LLW and LLC are respectively obtained by adding structural weakening and structural contraction:

$$
\frac{\Gamma \vdash B}{\Gamma, A \vdash B} \mathrm{~W} \quad \frac{\Gamma, A, A \vdash B}{\Gamma, A \vdash B} \mathrm{C}
$$

As with the sequent calculi for LL, LLW, and LLC, the intuitionistic calculi for ILZ, ILZW, and ILZC enjoy cut elimination and the subformula property for cut-free proofs.

2.2.3. Relevance Logic. The sequent calculus LR+ considered by Urquhart [30] for a fragment of relevance logic is IMALLC without $T$, i.e. ILZC restricted to $\{\multimap, \otimes, \mathbf{1}, \oplus, \&\}$.

\section{Alternating Branching VASS}

We define a "tree" extension of vector addition systems with states (VASS) that combines two kinds of branching behaviours: those of alternating VASS \$3.3.1) and those of branching VASS \$3.3.2. With this combination, we obtain a reformulation of Kopylov's vector addition games [14, for which he showed that

(1) the game is inter-reducible with LL provability

(2) the "lossy" version of the game is inter-reducible with LLW provability.

We further add full zero tests to this model, as they make the reduction from LL provability straightforward (see Section 4 ) and can easily be removed (see $\S 3.3 .3$.

\subsection{Definitions.}

3.1.1. Syntax. An alternating branching vector addition system with states and full zero tests $\left(\mathrm{ABVASS}_{\overline{0}}\right)$ is syntactically a tuple $\mathcal{A}=\left\langle Q, d, T_{u}, T_{f}, T_{s}, T_{z}\right\rangle$ where $Q$ is a finite set of states, $d$ is a dimension in $\mathbb{N}$, and $T_{u} \subseteq Q \times \mathbb{Z}^{d} \times Q$, $T_{f} \subseteq Q^{3}, T_{s} \subseteq Q^{3}$ and $T_{z} \subseteq Q^{2}$ are respectively finite sets of unary, fork, split and full zero test rules. We denote unary rules $\left(q, \overline{\mathrm{u}}, q_{1}\right)$ in $T_{u}$ with $\overline{\mathrm{u}}$ in $\mathbb{Z}^{d}$ by " $q \stackrel{\bar{u}}{\rightarrow} q_{1}$ ", fork rules $\left(q, q_{1}, q_{2}\right)$ in $T_{f}$ by " $q \rightarrow q_{1} \wedge q_{2}$ ", split rules $\left(q, q_{1}, q_{2}\right)$ in $T_{s}$ by " $q \rightarrow q_{1}+q_{2}$ ", and full zero test rules $\left(q, q_{1}\right)$ in $T_{z}$ by " $q \stackrel{\stackrel{?}{=} \overline{0}}{\longrightarrow} q_{1} "$.

3.1.2. Deduction Semantics. Given an ABVASS $_{\overline{0}}$, its semantics is defined by a deduction system over configurations $(q, \overline{\mathrm{v}})$ in $Q \times \mathbb{N}^{d}$ :

$$
\frac{q, \bar{v}}{q_{1}, \overline{\mathrm{v}}+\overline{\mathrm{u}}} \text { unary }
$$

where "+" denotes component-wise addition in $\mathbb{N}^{d}$, if $q \stackrel{\bar{u}}{\rightarrow} q_{1}$ is a rule (and implicitly $\bar{v}+\bar{u}$ has no negative component, i.e. is in $\mathbb{N}^{d}$ ), and

$$
\frac{q, \overline{\mathrm{v}}}{q_{1}, \overline{\mathrm{v}} q_{2}, \overline{\mathrm{v}}} \text { fork } \quad \frac{q, \overline{\mathrm{v}}_{1}+\overline{\mathrm{v}}_{2}}{q_{1}, \overline{\mathrm{v}}_{1} q_{2}, \overline{\mathrm{v}}_{2}} \text { split } \quad \frac{q, \overline{0}}{q_{1}, \overline{0}} \text { full-zero }
$$




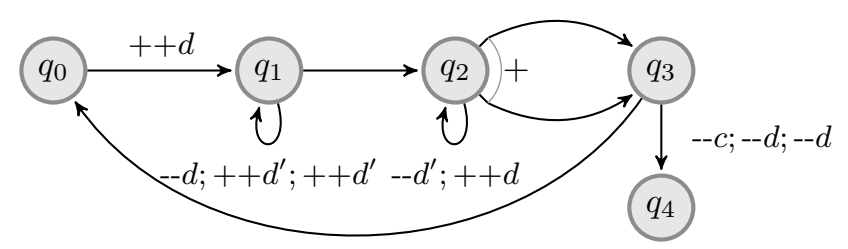

Figure 1. An example BVASS.

if $q \rightarrow q_{1} \wedge q_{2}, q \rightarrow q_{1}+q_{2}$, and $q \stackrel{\stackrel{?}{=} \overline{0}}{\longrightarrow} q_{1}$ are rules of the system, respectively, and " $\overline{0}$ " denotes the $d$-vector $\langle 0, \ldots, 0\rangle$ with zeroes on every coordinate. Such a deduction system can be employed either top-down or bottom-up depending on the decision problem at hand (as with tree automata); the top-down direction will correspond in a natural way to proof search in propositional linear logic, i.e. will correspond to the consequence to premises direction in the sequent calculus of $\$ 2.1 .2$.

3.1.3. Example. Let $\mathcal{A}$ be an ABVASS $_{\overline{0}}$ with five states $\left(q_{0}, q_{1}, q_{2}, q_{3}, q_{4}\right)$, of dimension 3 , with six unary rules:

$$
\begin{array}{lll}
q_{0} \stackrel{\langle 0,1,0\rangle}{\longrightarrow} q_{1} & q_{1} \stackrel{\langle 0,-1,2\rangle}{\longrightarrow} q_{1} & q_{1} \stackrel{\langle 0,0,0\rangle}{\longrightarrow} q_{2} \\
q_{2} \stackrel{\langle 0,1,-1\rangle}{\longrightarrow} q_{2} & q_{3} \stackrel{\langle 0,0,0\rangle}{\longrightarrow} q_{0} & q_{3} \stackrel{\langle-1,-2,0\rangle}{\longrightarrow} q_{4},
\end{array}
$$

and with one split rule $q_{2} \rightarrow q_{3}+q_{3}$. There are no fork rules and no full zero test rules in $\mathcal{A}$, and so it is a BVASS (see 33.3 .2 . A depiction of $\mathcal{A}$ is in Figure 1, where we write $c, d, d^{\prime}$ for vector indices $1,2,3$ (respectively), and specify unary rules in terms of increments and decrements.

From state $q_{0}$ and with $c, d, d^{\prime}$ initialised to 5,0,0 (i.e., from a root node labelled by $\left.\left(q_{0},\langle 5,0,0\rangle\right)\right), \mathcal{A}$ can reach $q_{2}$ with $d, d^{\prime}$ having values 2,0 , perform the split rule by dividing $c$ and $d$ almost equally (i.e., branch to two nodes labelled by $\left(q_{3},\langle 3,1,0\rangle\right)$ and $\left.\left(q_{3},\langle 2,1,0\rangle\right)\right)$, then in both threads reach $q_{2}$ again with $d, d^{\prime}$ having values 4,0 , perform the split rule as before, and finally reach $q_{4}$ with $c, d, d^{\prime}$ having values $1,0,0$ in one thread and 0,0,0 in the remaining three threads. See Figure 2 for the corresponding deduction tree.

Further reasoning, where we need to consider arbitrarily unequal splits, can show that $\mathcal{A}$ has a deduction tree whose root is labelled by $\left(q_{0},\langle m, 0,0\rangle\right)$ and with the state label at every leaf being $q_{4}$ if and only if $m \geq 4$. In fact, $\mathcal{A}$ is a slightly simplified version of the BVASS $\mathcal{B}_{2}$ in Section 6.

3.2. Decision Problems. Given an $\operatorname{ABVASS}_{\overline{0}} \mathcal{A}$ and a finite set of states $Q_{\ell}$, we denote by a root judgement " $\mathcal{A}, Q_{\ell} \triangleright q, \overline{\mathrm{v}}$ " the fact that there exists a deduction tree $\mathcal{D}$ in $\mathcal{A}$ with root label $(q, \bar{v})$ and leaf labels in $Q_{\ell} \times\{\overline{0}\}$. We call $\mathcal{D}$ a reachability witness for $(q, \bar{v})$. Root judgements can be derived through the following deduction rules, which will be handy in proofs:

$$
\begin{aligned}
& \overline{\mathcal{A}, Q_{\ell} \triangleright q_{\ell}, \overline{0}} \text { if } q_{\ell} \in Q_{\ell} \quad \frac{\mathcal{A}, Q_{\ell} \triangleright q_{1}, \overline{\mathrm{v}}+\overline{\mathrm{u}}}{\mathcal{A}, Q_{\ell} \triangleright q, \overline{\mathrm{v}}} \text { if } q \stackrel{\overline{\mathrm{u}}}{\rightarrow} q_{1} \quad \frac{\mathcal{A}, Q_{\ell} \triangleright q_{1}, \overline{0}}{\mathcal{A}, Q_{\ell} \triangleright q, \overline{0}} \text { if } q \stackrel{\stackrel{?}{\mathrm{D}} \overline{0}}{\rightarrow} q_{1} \\
& \frac{\mathcal{A}, Q_{\ell} \triangleright q_{1}, \overline{\mathrm{v}} \quad \mathcal{A}, Q_{\ell} \triangleright q_{2}, \overline{\mathrm{v}}}{\mathcal{A}, Q_{\ell} \triangleright q, \overline{\mathrm{v}}} \text { if } q \rightarrow q_{1} \wedge q_{2} \quad \frac{\mathcal{A}, Q_{\ell} \triangleright q_{1}, \overline{\mathrm{v}}_{1} \quad \mathcal{A}, Q_{\ell} \triangleright q_{2}, \overline{\mathrm{v}}_{2}}{\mathcal{A}, Q_{\ell} \triangleright q, \overline{\mathrm{v}}_{1}+\overline{\mathrm{v}}_{2}} \text { if } q \rightarrow q_{1}+q_{2}
\end{aligned}
$$




$$
\begin{array}{ll} 
& \frac{q_{0},\langle 5,0,0\rangle}{q_{1},\langle 5,1,0\rangle} \frac{}{q_{1},\langle 5,0,2\rangle} \\
& \frac{\frac{q_{2},\langle 5,0,2\rangle}{q_{2},\langle 5,1,1\rangle}}{q_{2},\langle 5,2,0\rangle} \\
\frac{\frac{q_{3},\langle 2,1,0\rangle}{q_{3},\langle 3,1,0\rangle}}{\frac{q_{0},\langle 3,1,0\rangle}{q_{1},\langle 3,2,0\rangle}} & \frac{\frac{q_{0},\langle 2,1,0\rangle}{q_{1},\langle 2,2,0\rangle}}{q_{1},\langle 3,1,2\rangle} \\
\frac{\frac{q_{1},\langle 2,1,2\rangle}{q_{2},\langle 3,0,4\rangle}}{\frac{q_{2},\langle 3,0,4\rangle}{q_{1},\langle 2,0,4\rangle}} & \frac{\frac{q_{2},\langle 2,0,4\rangle}{q_{2},\langle 2,1,3\rangle}}{q_{2},\langle 3,2,2,2,2\rangle} \\
\frac{\frac{q_{2},\langle 3,3,1\rangle}{q_{2},\langle 3,4,0\rangle}}{q_{3},\langle 2,2,0\rangle} & \frac{\frac{q_{3},\langle 1,2,0\rangle}{q_{2},\langle 2,3,1\rangle}}{q_{2},\langle 2,4,0\rangle} \\
\hline q_{4},\langle 1,0,0\rangle & \frac{q_{3},\langle 1,2,0\rangle}{q_{4},\langle 0,0,0\rangle} \quad \frac{q_{3},\langle 1,2,0\rangle}{q_{4},\langle 0,0,0\rangle}
\end{array}
$$

Figure 2. A deduction tree in the BVASS of Figure 1.

3.2.1. Reachability. Given an $\operatorname{ABVASS}_{\overline{0}} \mathcal{A}$, a finite set of states $Q_{\ell}$, and a state $q_{r}$, the reachability problem asks whether $\mathcal{A}, Q_{\ell} \triangleright q_{r}, \overline{0}$; we call a reachability witness for $\left(q_{r}, \overline{0}\right)$ more simply a reachability witness.

We will see in Section 4 that this reachability problem is equivalent to provability in LL; the problem is also related to games played over vectors of natural numbers, see Section 3.5. It is however undecidable:

Fact 3.1. Reachability in $A B V A S S_{\overline{0}}$ is undecidable.

Proof. Reachability is already undecidable in the more restricted model of AVASS, see Fact 3.2 below.

3.2.2. Lossy Reachability. In order to obtain decidability, we must weaken the ABVASS $_{\overline{0}}$ model or the decision problem. For the former, let us denote by $\overline{\mathrm{e}}_{i}$ the unit vector in $\mathbb{N}^{d}$ with one on coordinate $i$ and zero everywhere else. Then a lossy ABVASS $_{\overline{0}}$ can be understood as featuring a rule $q \stackrel{-\overline{\mathrm{e}}_{i}}{\longrightarrow} q$ for every $q$ in $Q$ and $0<i \leq d$. We rather define it by extending its deduction system with

$$
\frac{q, \overline{\mathrm{v}}}{q, \overline{\mathrm{v}}-\overline{\mathrm{e}}_{i}} \operatorname{loss}
$$

for every $q$ in $Q$ and $0<i \leq d$. We write ' $\nabla_{\ell}$ ' for root judgements where losses can occur. In terms of proof search in linear logic, losses will correspond to structural weakening, which is the distinguishing feature of affine linear logic.

Top-Down Coverability. An alternative way to see the reachability problem in lossy $\mathrm{ABVASS}_{\overline{0}}$ is to weaken the problem. Let us define a variant of ABVASS $_{\overline{0}}$ that feature full resets instead of full zero tests: we denote in this 
case rules $\left(q, q_{1}\right)$ in $T_{z}$ by $q \stackrel{:=\overline{0}}{\longrightarrow} q_{1}$ and associate a different semantics:

$$
\frac{q, \bar{v}}{q_{1}, \overline{0}} \text { full-reset }
$$

We call the resulting model ABVASSr. Given an ABVASSr $\mathcal{A}$, a state $q_{r}$, and a finite set of states $Q_{\ell}$, the top-down coverability or leaf coverability problem asks whether there exists a deduction tree $\mathcal{D}$ with root label $\left(q_{r}, \overline{0}\right)$ and such that, for each leaf, there exists some $q_{\ell}$ in $Q_{\ell}$ and some $\bar{v}$ in $\mathbb{N}^{d}$ such that the leaf label is $\left(q_{\ell}, \bar{v}\right)$; we then call $\mathcal{D}$ a coverability witness.

The reachability problem for lossy ABVASS $_{\overline{0}}$ is then equivalent to topdown coverability for ABVASSr. Observe indeed that the unary, fork, and split rules are monotone: if $\bar{v} \leq \bar{w}$ for the product ordering, i.e. if $\bar{v}(i) \leq \bar{w}(i)$ for all $0<i \leq d$, and a configuration $(q, \bar{v})$ allows to apply a rule and result in some configurations $\left(q_{1}, \overline{\mathbf{v}}_{1}\right)$ and (possibly) $\left(q_{2}, \overline{\mathrm{v}}_{2}\right)$, then $(q, \overline{\mathrm{w}})$ allows to apply the same rule and to obtain some $\left(q_{1}, \overline{\mathrm{w}}_{1}\right)$ and $\left(q_{2}, \overline{\mathrm{w}}_{2}\right)$ with $\overline{\mathrm{v}}_{1} \leq \overline{\mathrm{w}}_{1}$ and $\overline{\mathrm{v}}_{2} \leq \overline{\mathrm{w}}_{2}$. This means that losses in an $\operatorname{ABVASS}_{\overline{0}}$ can be applied as late as possible, either right before a full zero test or at the leaves-which corresponds exactly to top-down coverability for ABVASSr.

3.2.3. Expansive Reachability. In order to model structural contractions during proof search, it is natural to consider another variant of ABVASS $_{\overline{0}}$ called expansive $\mathrm{ABVASS}_{\overline{0}}$ and equipped with the deduction rules

$$
\frac{q, \overline{\mathrm{v}}+\overline{\mathrm{e}}_{i}}{q, \overline{\mathrm{v}}+2 \overline{\mathrm{e}}_{i}} \text { expansion }
$$

for every $q$ in $Q$ and $0<i \leq d$. We write ' $\triangleright_{e}$ ' for root judgements where expansions can occur. This is a restriction over ABVASS $_{\overline{0}}$ since expansions can be emulated through two unary rules $q \stackrel{-\overline{\mathrm{e}}_{i}}{\longrightarrow} q^{\prime} \stackrel{2 \overline{\mathrm{e}}_{i}}{\longrightarrow} q$. Expansive reachability is not quite dual to lossy reachability - we deal with increasing reachability in Section 7.

3.3. Restrictions. Note that $\mathrm{ABVASS}_{\overline{0}}$ generalise vector addition systems with states (VASS), which are ABVASS $_{\overline{0}}$ with only unary rules. They also generalise two "branching" extensions of VASS, which have been defined in relation with propositional linear logic. Since these restrictions do not feature full zero tests, their lossy reachability problem is equivalent to their top-down coverability problem.

3.3.1. Alternating VASS. were originally called "and-branching" counter machines by Lincoln et al. [18], and were introduced to prove the undecidability of propositional linear logic. Formally, an AVASS is an ABVASS $_{\overline{0}}$ which only features unary and fork rules, i.e. with $T_{s}=T_{z}=\emptyset$.

Fact 3.2 (Lincoln et al. [18]). Reachability in AVASS is undecidable.

Proof Idea. By a reduction from the halting problem in Minsky machines: note that a zero test $q \stackrel{c^{\prime}}{\longrightarrow} q^{\prime}$ on a counter $c$ can be emulated through a fork $q \rightarrow q^{\prime} \wedge q_{c}$, where unary rules $q_{c} \stackrel{-\overline{\mathrm{e}}_{c^{\prime}}}{\longrightarrow} q_{c}$ for all $c^{\prime} \neq c$ allow to empty the counters different from $c$, and a last unary rule $q_{c} \stackrel{\overline{0}}{\rightarrow} q_{\ell}$ to the single target state allows to check that $c$ was indeed equal to zero. 
Alternating VASS do not allow to model LL proof search in full; Kanovich [13 identified the matching LL fragment, called the $(!, \oplus)$-Horn fragment.

The complexity of the other basic reachability problems on AVASS is known:

- motivated by the complexity of fragments of relevance logic, Urquhart [30] proved that expansive reachability is complete for Ackermannian time, and

- motivated by the complexity of vector addition games (see Section 3.5), Courtois and Schmitz [8] showed that lossy reachability is 2-EXPTIME-complete.

3.3.2. Branching VASS. Inspired by the correspondences between the !-Horn fragment of linear logic and VASS unearthed by Kanovich [13, de Groote et al. 9] defined BVASS - which they originally dubbed "vector addition tree automata" - as a model of counter machines that matches MELL. Formally, a BVASS is an ABVASS $_{\overline{0}}$ with only unary and split rules, i.e. with $T_{f}=T_{z}=\emptyset$. This model turned out to be equivalent to independently defined models in linguistics [24] and protocol verification [32]; see [25] for a survey.

Whether BVASS reachability is decidable is an open problem, and is inter-reducible with MELL provability. Lazić [17 proved the best known lower bound to this day, which is 2-ExPSPACE-hardness. Two related problems were shown to be 2-ExpTime-complete by Demri et al. [10], namely increasing reachability (see Section 7) and boundedness.

3.3.3. Alternating Branching VASS. Kopylov [14] defined a one-player vector game, which matches essentially the reachability problem in ABVASS, i.e. in ABVASS $_{\overline{0}}$ with $T_{z}=\emptyset$. The elementary fragment of ILL defined by Larchey-Wendling and Galmiche [16] is another counterpart to ABVASS.

While allowing full zero tests is helpful in the reduction from LL provability, they can be dispensed with at little expense. Let us first introduce some notation. If node $n$ is an ancestor of a node $n^{\prime}$ in a deduction tree $\mathcal{D}$, and the labels of $n$ and $n^{\prime}$ are the same, we write $\mathcal{D}\left[n \leftarrow n^{\prime}\right]$ for the shortening of $\mathcal{D}$ obtained by replacing the subtree of rule applications rooted at $n$ by the one rooted at $n^{\prime}$. Observe that, if $\mathcal{D}$ is a reachability witness (resp. a coverability witness), then $\mathcal{D}\left[n \leftarrow n^{\prime}\right]$ is also a reachability witness (resp. a coverability witness).

Lemma 3.3. There is a logarithmic-space reduction from (lossy, resp. expansive) $A B V A S S_{\overline{0}}$ reachability to (lossy, resp. expansive) ABVASS reachability.

Proof. Suppose $\mathcal{A}$ is an $\operatorname{ABVASS}_{\overline{0}}$ with set of states $Q$ and dimension $d$.

For a logarithmic-space many-one reduction, the key observation is that, if there exists a witness for an instance of (lossy, resp. expansive) reachability for $\mathcal{A}$, then by repeated shortenings, there must be one in which, along every vertical path, the number of occurrences of full zero tests is at most $|Q|-1$.

It therefore suffices to decide the problem for an ABVASS $\mathcal{A}^{\dagger}$ whose set of states is $\{1, \ldots,|Q|\} \times Q$, whose dimension is $|Q| \cdot d$, and which simulates $\mathcal{A}$ up to $|Q|-1$ full zero tests along any vertical path. In any state $(i, q), \mathcal{A}^{\dagger}$ 
behaves like $\mathcal{A}$ in state $q$, but using the $i$ th $d$-tuple of its vector components.

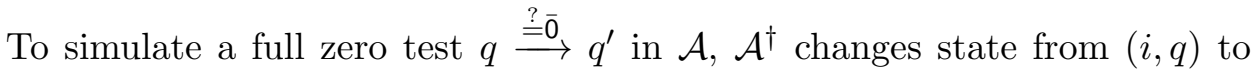
$(i+1, q)$, postponing the check that the $i$ th $d$-tuple of vector components are zero until the leaves of the deduction tree.

Remark 3.4 (Polynomial Time Turing Reduction). There is a polynomial time Turing reduction to the same effect. Its interest is that it preserves the dimension of the $\mathrm{ABVASS}_{\overline{0}}$. Because the dimension is - by far - the most important source of complexity in our upper bounds, preserving it might be useful in some circumstances.

Let us first define the set of root states relative to a subset $X$ of $Q$ by

$$
\operatorname{Root}_{\mathcal{A}}(X) \stackrel{\text { def }}{=}\{q \in Q \mid \mathcal{A}, X \triangleright q, \overline{0}\}
$$

as the set of states $q$ such that there exists a deduction in $\mathcal{A}$ with root label $(q, \overline{0})$ and leaf labels in $X \times\{\overline{0}\}$. The (lossy, resp. expansive) reachability problem for $\left\langle\mathcal{A}, q_{r}, Q_{\ell}\right\rangle$ then reduces to checking whether $q_{r}$ belongs to $\operatorname{Root}_{\mathcal{A}}\left(Q_{\ell}\right)$.

Let $\mathcal{A}=\left\langle Q, d, T_{u}, T_{f}, T_{s}, T_{z}\right\rangle$. Writing $\mathcal{A}^{\prime}$ for the ABVASS $\left\langle Q, d, T_{u}, T_{f}, T_{s}, \emptyset\right\rangle$, we can compute $\operatorname{Root}_{\mathcal{A}^{\prime}}(X)$ using $|Q|$ calls to an oracle for (lossy, resp. expansive) ABVASS reachability. Moreover, since $\operatorname{Root}_{\mathcal{A}^{\prime}}(X) \supseteq X$ is monotone, we can use a least fixed point computation that discovers root states according to the number of full zero tests along the branches of their reachability witnesses:

$$
\operatorname{Root}_{\mathcal{A}}\left(Q_{\ell}\right)=\mu X \operatorname{Root}_{\mathcal{A}^{\prime}}\left(Q_{\ell}\right) \cup \operatorname{Root}_{\mathcal{A}^{\prime}}\left(X \cup T_{z}^{-1}(X)\right) .
$$

This computation converges after at most $|Q|$ steps, and therefore works in polynomial time relative to the same oracle.

\subsection{Computational Complexity.}

3.4.1. Non-Elementary Complexity Classes. We will use in this paper two complexity classes [see 26]:

$$
\text { TOWER } \stackrel{\text { def }}{=} \bigcup_{e \in \text { FELEM }} \operatorname{DTIME}(\operatorname{tower}(e(n)))
$$

is the class of problems that can be solved with a deterministic Turing machine in time tower of some elementary function $e$ of the input, where tower $(0) \stackrel{\text { def }}{=} 1$ and tower $(n+1) \stackrel{\text { def }}{=} 2^{\text {tower }(n)}$ defines towers of exponentials. Similarly,

$$
\text { ACKERMANN } \stackrel{\text { def }}{=} \bigcup_{p \in \text { FPR }} \operatorname{DTime}(\operatorname{Ack}(p(n)))
$$

is the class of problems solvable in time Ack of some primitive recursive function $p$ of the input size, where "Ack" denotes the Ackermann functionany standard definition of Ack yields the same complexity class [26].

Completeness for TOWER is understood relative to many-one elementary reductions, and completeness for ACKERMANN relative to many-one primitive-recursive reductions. 
3.4.2. $A B V A S S_{0}^{-}$Complexity. For a set $T_{u}$ of unary rules, we write $\max ^{-}\left(T_{u}\right)$ (resp. $\max ^{+}\left(T_{u}\right)$ ) for the largest absolute value of any negative (resp. positive) integer in a vector in $T_{u}$, and $\max \left(T_{u}\right)$ for their overall maximum. We assume a binary encoding of the vectors in unary rules, thus $\max \left(T_{u}\right)$ might be exponential in the size of the $\mathrm{ABVASS}_{\overline{0}}$. We can however reduce to ordinary ABVASS $_{\overline{0}}$, i.e. ABVASS $_{\overline{0}}$ with $\overline{\mathrm{u}}=\overline{\mathrm{e}}_{i}$ or $\overline{\mathrm{u}}=-\overline{\mathrm{e}}_{i}$ for some $0<i \leq d$ whenever $q \stackrel{\overline{\mathrm{u}}}{\rightarrow} q_{1}$ is a unary rule:

Lemma 3.5. There is a logarithmic space reduction from reachability in (lossy, resp. expansive) $A B V A S S_{\overline{0}}$ to reachability in (lossy, resp. expansive) ordinary $A B V A S S_{\overline{0}}$.

Proof Idea. The idea is to encode each of the $d$ coordinates of the original ABVASS $_{\overline{0}}$ into $\left\lfloor\log \left(\max \left(T_{u}\right)+1\right)\right\rfloor$ coordinates, and each unary rule to apply a binary encoding of $\bar{u}$ to those new coordinates; see for instance [25] where this construction is detailed for BVASS. The expansive case requires to first explicitly encode expansions as unary rules.

Lossy Case. One of the main results of this paper is the following:

Theorem 3.6. Reachability in lossy BVASS and lossy ABVASS $S_{\overline{0}}$ is TOWERcomplete.

Proof. The upper bound is proved in Section 5. We present the hardness proof in detail in Section 6 .

Note that Theorem 3.6 entails an improvement for BVASS reachability over the 2-ExpSPACE lower bound of Lazić [17].

Expansive Case. Regarding expansive ABVASS $_{\overline{0}}$, we can adapt the proofs of Urquhart [30] for expansive AVASS and the relevance calculus LR+ to show:

Theorem 3.7. Reachability in expansive AVASS and expansive $A B V A S S_{\overline{0}}$ is ACKeRMANn-complete.

Proof. The lower bound is due to Urquhart [30, who proved hardness of expansive AVASS reachability by a direct reduction from the halting problem of Minsky machines with counter values bounded by the Ackermann function. The upper bound can be proved following essentially the same arguments as Urquhart's for LR+, using length function theorems for Dickson's Lemma [see e.g. 12]. See Section 7 for a proof.

Theorem 3.7 allows to derive the same ACKERMann bounds for provability in MALLC and LLC, see Section 7.

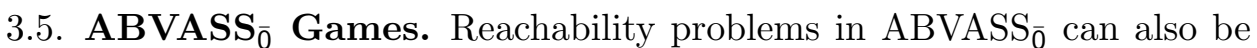
understood in a game-theoretic setting. Let us fix a reachability instance $\left\langle\mathcal{A}, q_{r}, Q_{\ell}\right\rangle$ and consider the following zero-sum two players game over the infinite arena $Q \times \mathbb{N}^{d}$, where $d$ is the dimension of $\mathcal{A}$ : its two players are called Controller and Environment. The game starts in the configuration $\left(q_{r}, \overline{0}\right)$. In a current configuration $(q, \bar{v})$, Controller chooses a rule of $\mathcal{A}$, which allows to apply one of the deduction rules of $\mathcal{A}$, or loses if none applies. In the case of a unary or full zero test rule, the current configuration changes to $\left(q_{1}, \bar{v}+\bar{u}\right)$ and $\left(q_{1}, \overline{0}\right)$ respectively. In the case of a fork rule, Environment 
chooses between a move to $\left(q_{1}, \bar{v}\right)$ or $\left(q_{2}, \bar{v}\right)$. In the case of a split rule, Controller furthermore chooses two vectors $\bar{v}_{1}, \bar{v}_{2}$ in $\mathbb{N}^{d}$ with $\bar{v}_{1}+\bar{v}_{2}=\bar{v}$ and Environment chooses between a move to $\left(q_{1}, \bar{v}_{1}\right)$ or a move to $\left(q_{2}, \bar{v}_{2}\right)$.

The objective of Controller is to reach a configuration $\left(q_{\ell}, \overline{0}\right)$ with $q_{\ell}$ in $Q_{\ell}$; the objective of Environment is to prevent it. It is easy to see that Controller has a winning strategy if and only if the original reachability instance was positive.

Increasing, expanding, or lossy reachability are straightforward to handle in this game setting. Interestingly, in the case of lossy reachability, we can take the full-reset semantics for $T_{z}$, and Controller's objective can then be restated as reaching $\left(q_{\ell}, \bar{v}\right)$ for some $q_{\ell}$ in $Q_{\ell}$ and some vector $\bar{v}$ in $\mathbb{N}^{d}$, i.e. as a state reachability objective. This game view is related to multi-dimensional energy games [5, 7, 1, which are played on AVASS (defined in \$3.3.1).

\section{Relationships Between LL and ABVASS $\overline{0}$}

4.1. From LL to $\mathbf{A B V A S S}_{\overline{0}}$. We proceed in two steps to show a reduction from LL provability to $\mathrm{ABVASS}_{\overline{0}}$ reachability: first, in $\$ 4.1 .1$, we recall a well-known reduction from LL provability to ILZ provability, and second, in $\$ 4.1 .2$, we exhibit a reduction from ILZ provability to ABVASS $_{\overline{0}}$ reachability. The outcome will thus be:

Proposition 4.1. There are polynomial space reductions:

(1) from (affine, resp. contractive) LL provability to (lossy, resp. expansive) $A B V A S S_{\overline{0}}$ reachability,

(2) from (affine, resp. contractive) MELL provability to (lossy, resp. expansive) $B V A S S_{\overline{0}}$ reachability.

4.1.1. From $L L$ to $I L Z$. The Kolmogorov translation of classical logic into intuitionistic logic by double negation can be adapted to linear logic [29]:

Fact 4.2. There is a polynomial time reduction from (affine, resp. contractive) LL provability to (affine, resp. contractive) ILZ provability.

Proof Idea. A translation of classical linear formulæ $A$ into intuitionistic ones $A^{k}$ is provided by Troelstra [29, Section 5.12], which satisfies

$$
\Gamma \vdash_{\mathrm{LL}} A \text { iff } \Gamma^{k} \vdash_{\mathrm{ILZ}} A^{k} .
$$

The proof of this fact uses in particular that, for all $A^{k}$,

$$
\vdash_{\text {ILZ }}\left(\left(A^{k} \multimap \perp\right) \multimap \perp\right) \circ A^{k} .
$$

We merely need to check that the result also holds in presence of structural weakenings or structural contractions. Because the sequent calculi for ILZW and ILZC are restrictions of those for LLW and LLC, we only need to exhibit a translation of the structural rules of the two-sided sequent calculus for LLW and LLC into ILZW and ILZC proofs.

For structural weakenings, the two-sided sequent calculus for LLW has rules

$$
\frac{\Gamma \vdash \Delta}{\Gamma, A \vdash \Delta} \mathrm{L}_{W} \quad \frac{\Gamma \vdash \Delta}{\Gamma \vdash A, \Delta} \mathrm{R}_{W}
$$

In order to prove the affine version of $(5)$, for $\left(\mathrm{L}_{W}\right)$ we need to restrict ourselves to $\Delta=B$ a single formula, and see that this is exactly the structural 
weakening of ILZW. For $\left(\mathrm{R}_{W}\right)$ we need to restrict ourselves to an empty $\Delta$ : then $\Gamma \vdash_{\text {LLW }}$ if and only if $\Gamma \vdash_{\text {LLW }} \perp$ and

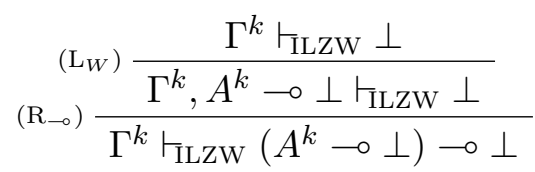

which, together with $\vdash_{\text {ILZW }}\left(\left(A^{k} \multimap \perp\right) \multimap \perp\right) \circ A^{k}$ for all $A^{k}$ by (6), allows to conclude.

For structural contractions, the two-sided sequent calculus for LLC has rules

$$
\frac{\Gamma, A, A \vdash \Delta}{\Gamma, A \vdash \Delta} \mathrm{L}_{C} \quad \frac{\Gamma \vdash A, A, \Delta}{\Gamma \vdash A, \Delta} \mathrm{R}_{C}
$$

Again, for $\left(\mathrm{L}_{C}\right)$ we have $\Delta=B$ a single formula and it turns out to be exactly the structural contraction of ILZC. For $\left(\mathrm{R}_{C}\right)$, necessarily $\Delta$ is empty. Then $\Gamma \vdash_{\text {LLC }} A, A$ if and only if $\Gamma, A \multimap \perp \vdash_{\text {LLC }} A$ and

$$
\begin{gathered}
\left(\mathrm{L}_{-}\right) \frac{\Gamma^{k}, A^{k} \multimap \perp \vdash_{\mathrm{ILZC}} A^{k} \overline{\perp \vdash_{\mathrm{ILZC}}}}{\left(\mathrm{L}_{\perp}\right)} \\
\left(\mathrm{R}_{\perp}\right) \frac{\Gamma^{k}, A^{k} \multimap \perp, A^{k} \multimap \perp \vdash_{\mathrm{LLZC}}}{\Gamma^{k}, A^{k} \multimap \perp, A^{k} \multimap \perp \vdash_{\mathrm{ILZC}} \perp} \\
\quad\left(\mathrm{L}_{C}\right) \frac{\Gamma^{k}, A^{k} \multimap \perp \vdash_{\mathrm{ILZC}} \perp}{\Gamma^{k} \vdash_{\mathrm{ILZC}}\left(A^{k} \multimap \perp\right) \multimap \perp}
\end{gathered}
$$

which, together with $\vdash_{\mathrm{LLCC}}\left(\left(A^{k} \multimap \perp\right) \multimap \perp\right) \circ A^{k}$ for all $A^{k}$ by $(6)$, allows to conclude.

4.1.2. From ILZ to $A B V A S S_{\overline{0}}$. The key property of the sequent calculi for ILZ, ILZW, and ILZC we exploit in our reduction to ABVASS $_{\overline{0}}$ is the subformula property of cut-free proofs.

Let us consider an instance of the provability problem for ILZ, i.e. some formula $F$. The subformula property allows us to view a sequent $! \Psi, \Delta \vdash A$ appearing in a cut-free proof of $\vdash F$ as a triple consisting of a multiset $! \Psi$ of !-guarded subformulæ, a multiset $\Delta$ of subformulæ, and a subformula $A$-all subformulæ of the target formula $F$. Thanks to logical weakening (!W) and logical contraction (!C), we will even be able to treat ! $\Psi$ as a set.

Let us write $S$ for the set of subformulæ of $F$ and $S ! \subseteq S$ for its !guarded subformulæ. We define from $F$ an $\operatorname{ABVASS}_{\overline{0}} \mathcal{A}_{F}^{I}$ of dimension $|S|$ that includes $2^{S !} \times(S \uplus\{\}$.$) in its state space, where "." is a fresh$ symbol. A configuration of $\mathcal{A}_{F}^{I}$ in $2^{S !} \times S \times \mathbb{N}^{S}$ encodes a sequent ! $\Psi, \Delta \vdash$ $A$ as $(\sigma(! \Psi), A, \Delta)$, where $\sigma$ associates to a multiset its support, i.e. its set without duplicates - note that we completely identify multisets in $\mathbb{N}^{S}$ with vectors in $\mathbb{N}^{|S|}$. A configuration in $\left(2^{S !} \times\{.\} \times \mathbb{N}^{S}\right)$ encodes a sequent $! \Psi, \Delta \vdash$ as $(\sigma(! \Psi), ., \Delta)$. We also include a distinguished leaf state $q_{\ell}$ in the state space of $\mathcal{A}_{F}^{I}$. The rules of $\mathcal{A}_{F}^{I}$ implement the rules of ILZ on the encoded configurations in a straightforward manner - they rely on additional intermediate states for this - and are depicted in Figure 3. An additional store rule allows to move an "of-course" ! A formula from counters to state storage; we could compile it into the other rules at the expense of a larger number of cases. 


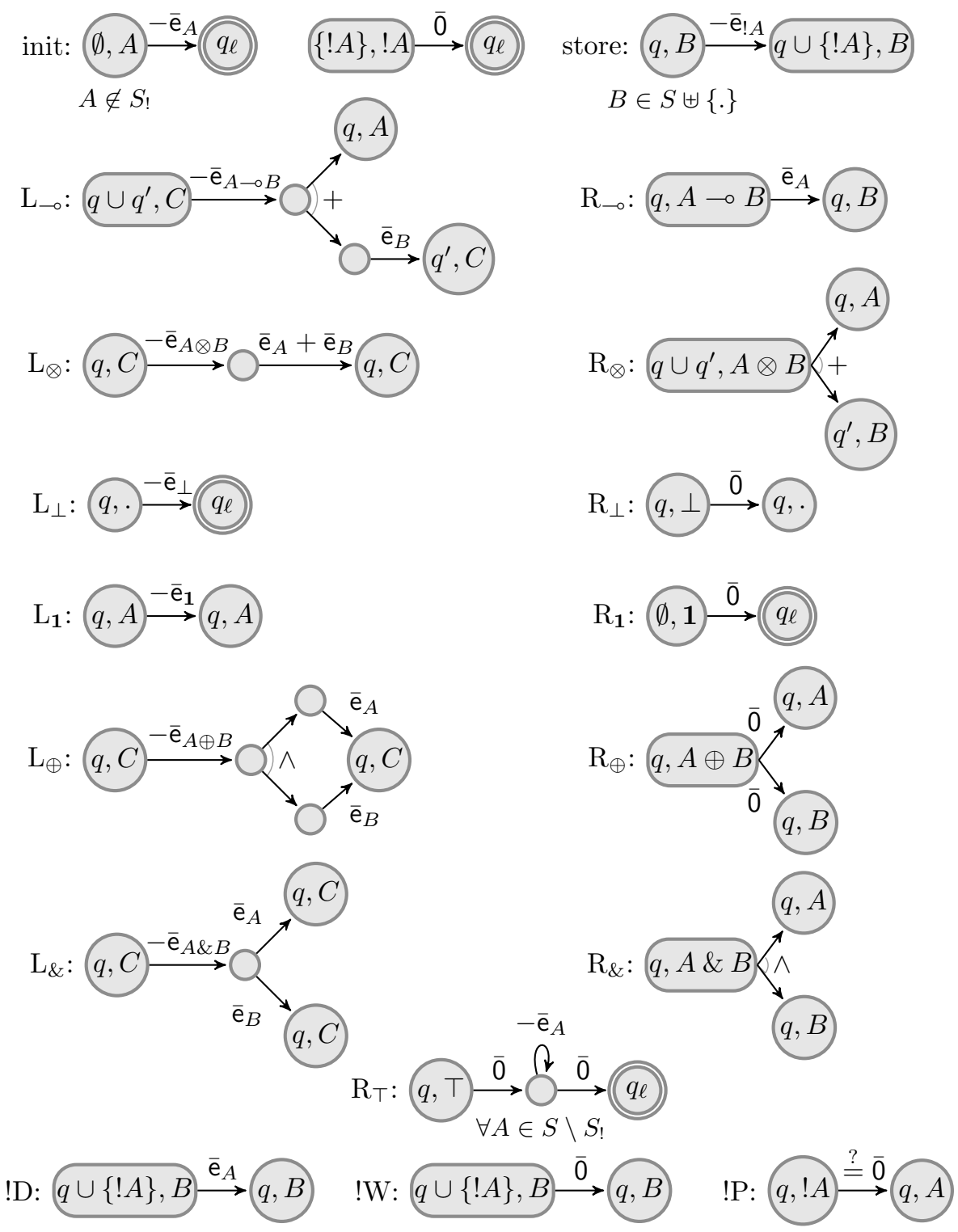

Figure 3 . The rules of $\mathcal{A}_{F}^{I} ; q, q^{\prime}$ are subsets of $S_{!}$, all the formulæ must be in $S$.

For $A$ empty or a formula in $S$, we write $A^{\dagger} \stackrel{\text { def }}{=}$. if $A$ is empty and $A^{\dagger} \stackrel{\text { def }}{=} A$ otherwise. The following claim relates ILZ proofs with deductions in $\mathcal{A}_{F}^{I}$ :

Claim 4.3.1 ( $\mathcal{A}_{F}^{I}$ is Sound and Complete). For all ! $\Psi$ in $\mathbb{N}^{S !}, \Delta$ in $\mathbb{N}^{S \backslash S !}$ and $A^{\dagger}$ in $S \uplus\{$.$\} ,$

$$
\mathcal{A}_{F}^{I},\left\{q_{\ell}\right\} \triangleright \sigma(! \Psi), A^{\dagger}, \Delta \quad \text { iff } \quad ! \Psi, \Delta \vdash_{\mathrm{ILZ}} A .
$$

Completeness Proof. Let us prove by induction on the height of a proof tree for $! \Psi, \Delta \vdash_{\text {ILZ }} A$ that $\mathcal{A}_{F}^{I},\left\{q_{\ell}\right\} \triangleright \sigma(! \Psi), A^{\dagger}, \Delta$. This boils down to a verification that the rules in Figure 3 implement the sequent calculus for 
ILZ. We will not detail all the cases, but here are four instances for (init), $\left(\mathrm{L}_{-}\right),\left(\mathrm{L}_{\oplus}\right)$, and $(! \mathrm{P})$ - the remaining cases being similar.

For (init): we know that $\mathcal{A}_{F}^{I},\left\{q_{\ell}\right\} \triangleright q_{\ell}, \overline{0}$ vacuously. We then distinguish two cases: either $A$ is not in $S_{!}$, and the first (init) unary rule applies and allows to deduce $\mathcal{A}_{F}^{I},\left\{q_{\ell}\right\} \triangleright \emptyset, A, \overline{\mathrm{e}}_{A}$ as desired, or $A$ is in $S_{1}$, and the second (init) unary rule applies and allows to deduce $\mathcal{A}_{F}^{I},\left\{q_{\ell}\right\} \triangleright\{A\}, A, \overline{0}$ as desired.

For $\left(\mathbf{L}_{-}\right)$: let us write $! \Phi, \Gamma \vdash_{\text {ILZ }} A$ and $! \Psi, \Delta, B \vdash_{\text {LLZ }} C$ for the premises with $! \Phi$ and $! \Psi$ in $\mathbb{N}^{S !}, \Gamma$ and $\Delta$ in $\mathbb{N}^{S \backslash S !}$, and $A, B, C$ in $S$. By induction hypothesis, $\mathcal{A}_{F}^{I},\left\{q_{\ell}\right\} \triangleright \sigma(! \Phi), A, \Gamma$. If $B$ is in $S_{!}$, then by induction hypothesis $\mathcal{A}_{F}^{I},\left\{q_{\ell}\right\} \triangleright \sigma(! \Psi) \cup B, C, \Delta$ and we can apply the store rule to show $\mathcal{A}_{F}^{I},\left\{q_{\ell}\right\} \triangleright \sigma(! \Phi), C, \Delta+\overline{\mathrm{e}}_{B}$. Otherwise, i.e. if $B$ is in $S \backslash S$ ! the induction hypothesis provides us with the same sequent directly. Applying the rules for $\left(\mathrm{L}_{-}\right)$, we see that $\mathcal{A}_{F}^{I},\left\{q_{\ell}\right\} \triangleright \sigma(! \Phi) \cup \sigma(! \Psi), C, \Gamma+\Delta+\overline{\mathrm{e}}_{A \multimap B}$ as desired.

For $\left(\mathbf{L}_{\oplus}\right)$ : let us write ! $\Phi, \Gamma, A \vdash_{\mathrm{ILZ}} C$ and $! \Phi, \Gamma, B \vdash_{\mathrm{ILZ}} C$ for the premises with ! $\Phi$ in $\mathbb{N}^{S !}, \Gamma$ in $\mathbb{N}^{S \backslash S !}$, and $A, B, C$ in $S$. Like in the previous argument for $\left(\mathrm{L}_{-}\right)$, we can use the store rule if $A$ or $B$ are in $S_{!}$, and in any case the induction hypothesis shows $\mathcal{A}_{F}^{I},\left\{q_{\ell}\right\} \triangleright \sigma(! \Phi), C, \Gamma+\overline{\mathrm{e}}_{A}$ and $\mathcal{A}_{F}^{I},\left\{q_{\ell}\right\} \triangleright \sigma(! \Phi), C, \Gamma+\overline{\mathrm{e}}_{B}$. The ABVASS $_{\overline{0}}$ rules for $\left(\mathrm{L}_{\oplus}\right)$ then allow to remove $\overline{\mathrm{e}}_{A}$ and $\overline{\mathrm{e}}_{B}$ from these two configurations, apply the fork rule, and finally add $\overline{\mathrm{e}}_{A \oplus B}$ to prove $\mathcal{A}_{F}^{I},\left\{q_{\ell}\right\} \triangleright \sigma(! \Phi), C, \Gamma+\overline{\mathrm{e}}_{A \oplus B}$ as desired.

For (!P): by induction hypothesis $\mathcal{A}_{F}^{I},\left\{q_{\ell}\right\} \triangleright \sigma(! \Gamma), A, \overline{0}$ and we can apply the corresponding full zero test to show $\mathcal{A}_{F}^{I},\left\{q_{\ell}\right\} \triangleright \sigma(! \Gamma), ! A, \overline{0}$ as desired.

Soundness Proof. As a preliminary observation, note that the store rule of $\mathcal{A}_{F}^{I}$ is the only rule that can decrement the counter of a formula from $S$. By induction over the height of deduction trees for $\mathcal{A}_{F}^{I}$, we can normalise deductions so that store rules are applied either immediately after a rule that added $\bar{e}_{! A}$ to the current configuration, or immediately at the root of the deduction (if $F$ itself is in $S_{!}$). This means that we can assume $\Delta$ in $\mathbb{N}^{S \backslash S !}$ in a root judgement $\mathcal{A}_{F}^{I},\left\{q_{\ell}\right\} \triangleright \sigma(! \Psi), A^{\dagger}, \Delta$ as in the statement of the claim.

Let us prove by induction on the height of a deduction tree for $\mathcal{A}_{F}^{I},\left\{q_{\ell}\right\} \triangleright$ $\sigma(! \Psi), A^{\dagger}, \Delta$ that $! \Psi, \Delta \vdash_{\text {ILz }} A$. Again, we only cover a few cases, depending on which group of rules of $\mathcal{A}_{F}^{I}$ was applied last:

For (init): either the first variant for $A$ in $S \backslash S$ ! was used to obtain $\mathcal{A}_{F}^{I},\left\{q_{\ell}\right\} \triangleright \emptyset, A, \overline{\mathrm{e}}_{A}$, and we have $A \vdash_{\mathrm{ILZ}} A$ as desired, or the second variant for $! A$ in $S$ ! was used to obtain $\mathcal{A}_{F}^{I},\left\{q_{\ell}\right\} \triangleright ! A, ! A, \overline{0}$, and we have $! A \vdash_{\mathrm{ILZ}} ! A$ as desired.

For $\left(\mathbf{L}_{-}\right)$: we know that $\mathcal{A}_{F}^{I},\left\{q_{\ell}\right\} \triangleright q, A, \Gamma$ and $\mathcal{A}_{F}^{I},\left\{q_{\ell}\right\} \triangleright q^{\prime}, C, \Delta+\overline{\mathrm{e}}_{B}$ for some $q$ and $q^{\prime}$ included in $S_{!}$, some $\Gamma$ and $\Delta$ in $\mathbb{N}^{S \backslash S !}$, and some $A, B$, and $C$ in $S$. By induction hypothesis, there exists ! $\Phi$ in $\mathbb{N}^{S !}$ with $q=\sigma(! \Phi)$ such that $! \Phi, \Gamma \vdash_{\text {ILZ }} A$. If $B$ is in $S !$-and is therefore the result of a store rule applied right after-, then $\mathcal{A}_{F}^{I},\left\{q_{\ell}\right\} \triangleright q^{\prime} \cup$ $\{B\}, C, \Delta$ and by induction hypothesis there exists $! \Psi$ in $\mathbb{N}^{S !}$ with 
$\sigma(! \Psi)=q^{\prime}$ such that $! \Psi, B, \Delta \vdash_{\text {LLz }} C$. Otherwise, i.e. if $B$ is in $S \backslash S !$ we obtain the latter sequent directly by induction hypothesis. Then $! \Psi, ! \Phi, B, \Delta, \Gamma, A \multimap B \vdash_{\mathrm{LLZ}} C$ as desired.

For $\left(\mathbf{L}_{\oplus}\right)$ : we know that $\mathcal{A}_{F}^{I},\left\{q_{\ell}\right\} \triangleright q, C, \Gamma+\overline{\mathrm{e}}_{A}$ and $\mathcal{A}_{F}^{I},\left\{q_{\ell}\right\} \triangleright q, C, \Gamma+$ $\overline{\mathrm{e}}_{B}$ for some $q$ included in $S_{!}$, some $\Gamma$ in $\mathbb{N}^{S \backslash S !}$, and some $A, B$, and $C$ in $S$. Using store rules, we can argue as in the case of $\left(\mathrm{L}_{-}\right)$ that, regardless of whether $A$ or $B$ is in $S_{!}$, by induction hypothesis there exists $! \Phi$ in $\mathbb{N}^{S !}$ with $q=\sigma(! \Phi)$ such that $! \Phi, A, \Gamma \vdash_{\text {ILZ }} C$ and $! \Phi, B, \Gamma \vdash_{\text {ILZ }} C$. Therefore $! \Phi, A \oplus B, \Gamma \vdash_{\text {ILZ }} C$ as desired.

For (!P): we know that $\mathcal{A}_{F}^{I},\left\{q_{\ell}\right\} \triangleright q, A, \overline{0}$, thus by induction hypothesis there exists $! \Psi$ in $\mathbb{N}^{S !}$ with $q=\sigma(! \Psi)$ and $! \Psi \vdash_{\text {ILZ }} A$, hence $! \Psi \vdash_{\mathrm{ILZ}} ! A$ as desired.

Claim 4.3.2 (Affine Case). When allowing losses in $\mathcal{A}_{F}^{I}$, for all ! $\Psi$ in $\mathbb{N}^{S !}, \Delta$ in $\mathbb{N}^{S \backslash S !}$ and $A^{\dagger}$ in $S \uplus\{$.$\} ,$

$$
\mathcal{A}_{F}^{I},\left\{q_{\ell}\right\} \triangleright_{\ell} \sigma(! \Psi), A^{\dagger}, \Delta \quad \text { iff } \quad ! \Psi, \Delta \vdash_{\mathrm{ILZW}} A .
$$

Proof. As a preliminary observation, note that, by monotonicity, losses occurring inside a group of rules depicted in Figure 3 can be delayed until after the execution of the group is completed. By Claim 4.3.1, it therefore suffices to check the case of the loss and structural weakening rules.

For completeness, if (W) is the last applied rule in a proof of $! \Psi, B, \Delta \vdash_{\text {ILZW }}$ $A$, then, by induction hypothesis $\mathcal{A}_{F}^{I},\left\{q_{\ell}\right\} \triangleright_{\ell} \sigma(! \Psi), A, \Delta$, and an application of the loss rule yields $\mathcal{A}_{F}^{I},\left\{q_{\ell}\right\} \triangleright_{\ell} \sigma(! \Psi), A, \Delta+\overline{\mathrm{e}}_{B}$ as desired.

For soundness, if a loss of some $B$ is the last applied rule in a deduction showing $\mathcal{A}_{F}^{I},\left\{q_{\ell}\right\} \triangleright_{\ell} q, A, \Delta+\overline{\mathrm{e}}_{B}$, then $\mathcal{A}_{F}^{I},\left\{q_{\ell}\right\} \triangleright_{\ell} q, A, \Delta$. By induction hypothesis there exists $! \Psi$ with $q=\sigma(! \Psi)$ such that $! \Psi \Delta \vdash_{\text {ILZW }} A$, from which (W) yields ! $\Psi, B, \Delta \vdash_{\text {ILZW }} A$ as desired.

Claim 4.3.3 (Contractive Case). When allowing expansions in $\mathcal{A}_{F}^{I}$, for all ! $\Psi$ in $\mathbb{N}^{S_{!}}, \Delta$ in $\mathbb{N}^{S \backslash S !}$ and $A^{\dagger}$ in $S \uplus\{$.$\} ,$

$$
\mathcal{A}_{F}^{I},\left\{q_{\ell}\right\} \triangleright_{e} \sigma(! \Psi), A^{\dagger}, \Delta \quad \text { iff } \quad ! \Psi, \Delta \vdash_{\mathrm{ILZC}} A .
$$

Proof. As a preliminary observation, note that, by monotonicity, expansions occurring inside a group of rules depicted in Figure 3 can be applied before the execution of the group is started. By Claim 4.3.1, it therefore suffices to check the case of the expansion and structural contraction rules.

For completeness, if (C) is the last applied rule in a proof of ! $\Psi, B, \Delta \vdash_{\mathrm{ILZC}}$ $A$, then we can assume that the contracted formula was some $B$ in $S \backslash S$ ! as otherwise logical weakening would have sufficed, thus ! $\Psi, B, B, \Delta \vdash_{\text {ILZW }} A$. By induction hypothesis, $\mathcal{A}_{F}^{I},\left\{q_{\ell}\right\} \triangleright_{e} \sigma(! \Psi), A, \Delta+2 \overline{\mathrm{e}}_{B}$, and an application of the expansion deduction rule yields $\mathcal{A}_{F}^{I},\left\{q_{\ell}\right\} \triangleright_{e} \sigma(! \Psi), A, \Delta$ as desired.

For soundness, assume that an expansion is the rule applied at the root of a deduction tree for $\mathcal{A}_{F}^{I},\left\{q_{\ell}\right\} \triangleright_{e} q, A, \Delta+\overline{\mathrm{e}}_{B}$, hence that $\mathcal{A}_{F}^{I},\left\{q_{\ell}\right\} \triangleright_{e}$ $q, A, \Delta+2 \overline{\mathrm{e}}_{B}$. Because we assume store rules to occur as early as possible, $B$ cannot be in $S_{!}$. Thus, by induction hypothesis there exists ! $\Psi$ with

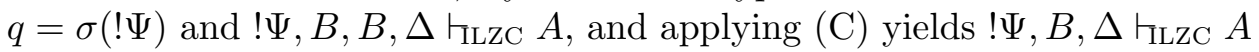
as desired.

Proposition 4.3. There are polynomial space reductions: 
(1) from (affine, resp. contractive) ILZ provability to (lossy, resp. expansive) $A B V A S S_{\overline{0}}$ reachability,

(2) from (affine, resp. contractive) IMELZ provability to (lossy, resp. expansive) $B V A S S_{\overline{0}}$ reachability.

Proof. For 1, we reduce the provability of $\vdash F$ to the reachability of $(\emptyset, F)$ in $\mathcal{A}_{F}^{I}$, which is correct thanks to the subformula property and claims 4.3.1. 4.3 .3 .

For 2 , simply observe that $\left(\mathrm{L}_{\oplus}\right)$ and $\left(\mathrm{R}_{\&}\right)$ are the only rules of ILZ that make use of fork rules in $\mathcal{A}_{F}^{I}$.

Our reductions incur an exponential blow-up in the number of stateshowever, as we will see with our complexity upper bounds, this is not an issue, because the main source of complexity in ABVASS $_{\overline{0}}$ is, by far, the dimension of the system, which is here linear in $|F|$.

4.2. From ABVASS ${ }_{\overline{0}}$ to LL. In order to exhibit a reduction from $\operatorname{ABVASS}_{\overline{0}}$ reachability to LL provability, we extend a similar reduction proved by Lincoln, Mitchell, Scedrov, and Shankar [18] in the case of AVASS (also employed by Urquhart [30]). The general idea is to encode ABVASS $_{\overline{0}}$ configurations as sequents and ABVASS $_{\overline{0}}$ deductions as proofs in LL extended with a theory, where encoded ABVASS $_{\overline{0}}$ rules are provided as an additional set of non-logical axioms.

4.2.1. Linear Logic with a Theory. In the framework of Lincoln et al., a theory $T$ is a finite set of axioms $C, p_{1}^{\perp}, \ldots, p_{m}^{\perp}$ where $C$ is a MALL formula and each $p_{i}$ is an atomic proposition. Proofs in LL $+T$ can employ two new rules

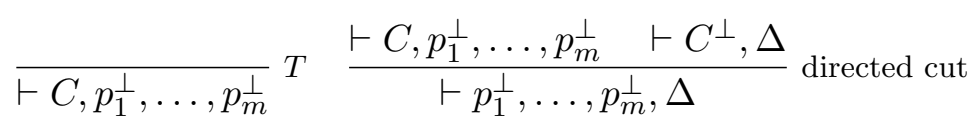

where $C, p_{1}^{\perp}, \ldots, p_{m}^{\perp}$ belongs to $T$.

A proof in LL+T is directed if all its cuts are directed cuts. By adapting the LL cut-elimination proof, Lincoln et al. show:

Fact $4.4([18])$. If there is a proof of $\vdash \Gamma$ in $L L+T$, then there is a directed proof of $\vdash \Gamma$ in $L L+T$.

The axioms of a theory $T$ can be translated in pure LL by

$$
\left\ulcorner C, p_{1}^{\perp}, \ldots, p_{m}^{\perp}\right\urcorner \stackrel{\text { def }}{=} C^{\perp} \otimes p_{1} \otimes \cdots \otimes p_{m} .
$$

Fact $4.5([18])$. For any finite set of axioms $T, \vdash \Gamma$ is provable in $L L+T$ if and only if $\vdash ?\ulcorner T\urcorner, \Gamma$ is provable in $L L$.

4.2.2. Encoding $A B V A S S_{\overline{0}}$. Given an ABVASS $\mathcal{A}=\left\langle Q, d, T_{u}, T_{f}, T_{s}, \emptyset\right\rangle$, a configuration $(q, \overline{\mathrm{v}})$ in $Q \times \mathbb{N}^{d}$ is encoded as the sequent

$$
\theta(q, \overline{\mathrm{v}}) \stackrel{\text { def }}{=} \vdash q^{\perp},\left(e_{1}^{\perp}\right)^{\overline{\mathrm{v}}(1)}, \ldots,\left(e_{d}^{\perp}\right)^{\overline{\mathrm{v}}(d)}
$$

where $Q \uplus\left\{e_{i} \mid i=1, \ldots, d\right\}$ is included in the set of atomic propositions and $A^{n}$ stands for the formula $A$ repeated $n$ times.

By Lemma 3.5 we assume $\mathcal{A}$ to be in ordinary form. We construct from the rules of $\mathcal{A}$ a theory $T$ consisting of sequents of form $\vdash q^{\perp}, c_{1}^{\perp}, \ldots, c_{m}^{\perp}, C$ 
with $q$ in $Q$ the originating state, $c_{j}$ in $\left\{e_{i} \mid 0<i \leq d\right\}$, and $C$ a MALL formula containing the destination state(s) positively. Here are the axioms corresponding to each type of rule:

$$
\begin{array}{ll}
q \stackrel{\overline{\mathrm{e}}_{i}}{\rightarrow} q_{1} & q^{\perp}, q_{1} \otimes e_{i} \\
q \stackrel{-\overline{\mathrm{e}}_{i}}{\rightarrow} q_{1} & q^{\perp}, e_{i}^{\perp}, q_{1} \\
q \rightarrow q_{1} \wedge q_{2} & q^{\perp}, q_{1} \oplus q_{2} \\
q \rightarrow q_{1}+q_{2} & q^{\perp}, q_{1}>q_{2}
\end{array}
$$

By Lemma 3.3, we do not need to consider the case of full zero tests. Here is nevertheless how they could be encoded, provided we slightly extended the reduction of LL+T to LL in Fact 4.5 to allow exponentials in $T$ :

$$
q \stackrel{\stackrel{?}{=} \overline{0}}{\longrightarrow} q_{1}
$$

Claim 4.6.1. For all $(q, \bar{v})$ in $Q \times \mathbb{N}^{d}, \mathcal{A}, Q_{\ell} \triangleright q, \bar{v}$ if and only if $\vdash \theta(q, \bar{v}), ? Q_{\ell}$ in $\mathrm{LL}+T$.

Proof. The AVASS case is proved by Lincoln et al. [18, Lemmata 3.5 and 3.6] by induction on the height of deduction trees in $\mathcal{A}$ and the number of directed cuts in a directed proof in LL $+T$ (with minor adaptations for ? $Q_{\ell}$ ). Thus, we only need to prove that split rules preserve this statement ${ }^{2}$

Assume for the direct implication that $\mathcal{A}, Q_{\ell} \triangleright q, \bar{v}$ as the result of a split rule $q \rightarrow q_{1}+q_{2}$, thus $\bar{v}=\bar{v}_{1}+\bar{v}_{2}$ and $\mathcal{A}, Q_{\ell} \triangleright q_{1}, \bar{v}_{1}$ and $\mathcal{A}, Q_{\ell} \triangleright q_{2}, \bar{v}_{2}$. By induction hypothesis, $\vdash \theta\left(q_{1}, \bar{v}_{1}\right), ? Q_{\ell}$ and $\vdash \theta\left(q_{2}, \bar{v}_{2}\right), ? Q_{\ell}$, and we have the following proof of $\vdash \theta(q, \bar{v}), ? Q_{\ell}$ :

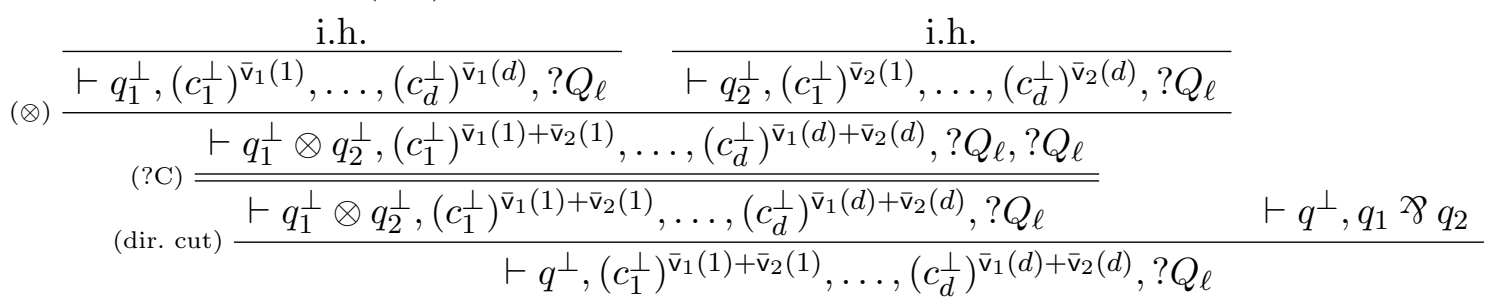

Conversely, assume that the last applied directed cut has

$$
\vdash q_{1}^{\perp} \otimes q_{2}^{\perp},\left(c_{1}^{\perp}\right)^{\bar{v}(1)}, \ldots,\left(c_{d}^{\perp}\right)^{\bar{v}(d)}, ? Q_{\ell}
$$

and $\vdash q^{\perp}, q_{1}>8 q_{2}$ as premises. The only rules that allow to prove (9) are $(? \mathrm{D}),(? \mathrm{C})$ or $(? \mathrm{~W})$ applied to some $q_{\ell}$ in $Q_{\ell}$, and $(\otimes)$. Logical contractions are irrelevant to the claim, and wlog. we can apply derelictions above $(\otimes)$, thus we know that $(9)$ is the result of $(\otimes)$ followed by a series of $($ ?W $)$. Hence $\vdash \theta\left(q_{1}, \overline{\mathrm{v}}_{1}\right), ? Q_{1}$ and $\vdash \theta\left(q_{2}, \overline{\mathrm{v}}_{2}\right), ? Q_{2}$ with $\overline{\mathrm{v}}=\overline{\mathrm{v}}_{1}+\overline{\mathrm{v}}_{2}$ and $Q_{\ell} \supseteq Q_{1} \cup Q_{2}$. By induction hypothesis, $\mathcal{A}, Q_{1} \triangleright q_{1}, \bar{v}_{1}$ and $\mathcal{A}, Q_{2} \triangleright q_{2}, \bar{v}_{2}$. Because $Q_{1} \subseteq Q_{\ell}$ and $Q_{2} \subseteq Q_{\ell}$ this entails $\mathcal{A}, Q_{\ell} \triangleright q_{1}, \overline{\mathrm{v}}_{1}$ and $\mathcal{A}, Q_{\ell} \triangleright q_{2}, \overline{\mathrm{v}}_{2}$, from which a split allows to derive $\mathcal{A}, Q_{\ell} \triangleright q, \overline{\mathrm{v}}$ as desired.

Proposition 4.6. There are logarithmic space reductions

(1) from $A B V A S S_{\overline{0}}$ reachability to LL provability and

(2) from $B V A S S_{\overline{0}}$ reachability to MELL provability.

\footnotetext{
${ }^{2}$ de Groote et al. 9] show how to handle split rules in IMELL, but they do not rely on the LL+T framework, which motivates considering this case here.
} 
Proof. By Lemma 3.3 we can eliminate full zero tests. For 1, by Claim 4.6.1 and Fact 4.5, $\mathcal{A}, Q_{\ell} \triangleright q_{r}, \overline{0}$ if and only if $\vdash q_{r}^{\perp}, ? Q_{\ell}, ?\ulcorner T\urcorner$. Regarding 2 simply observe that additive connectives are only used for the encoding of fork rules.

4.2.3. Affine Case. Adapting the proof of Proposition 4.6 to the affine case is relatively straightforward. For starters, Fact 4.4 also holds for LLW $+T$ using the cut elimination procedure for LLW, and allowing structural weakenings does not influence the proof of Fact 4.5 in [18, Lemmata 3.2 and 3.3]. We show:

Proposition 4.7. There are logarithmic space reductions

(1) from $A B V A S S_{\overline{0}}$ lossy reachability to LLW provability and

(2) from $B V A S S_{\overline{0}}$ lossy reachability to MELLW provability.

This relies on an extension of Claim 4.6.1.

Claim 4.7.1. For all $(q, \bar{v})$ in $Q \times \mathbb{N}^{d}, \mathcal{A}, Q_{\ell} \triangleright_{\ell} q, \bar{v}$ with lossy semantics if and only if $\vdash \theta(q, \bar{v}), ? Q_{\ell}$ in $L L W+T$.

Proof. We proceed as before by induction on the height of a deduction tree in $\mathcal{A}$ and on the number of directed cuts in a proof in LLW $+T$. The only new cases to consider in addition to those of Claim 4.6.1 are those of losses and structural weakenings. In case of a loss allowing to derive $\mathcal{A}, Q_{\ell} \triangleright_{\ell}$ $q, \overline{\mathrm{v}}+\overline{\mathrm{e}}_{i}$, by induction hypothesis $\vdash \theta(q, \overline{\mathrm{v}}), ? Q_{\ell}$ and a structural weakening yields $\vdash \theta\left(q, \overline{\mathrm{v}}+\overline{\mathrm{e}}_{i}\right), ? Q_{\ell}$ as desired. Conversely, in case of a structural weakening allowing to derive $\vdash \theta(q, \overline{\mathrm{v}}), ? Q_{\ell}$, either the weakened formula is some ? $q_{\ell}$ guarded by ? and by induction hypothesis $\mathcal{A}, Q_{\ell} \backslash\left\{q_{\ell}\right\} \triangleright_{\ell} q, \bar{v}$ thus $\mathcal{A}, Q_{\ell} \triangleright_{\ell} q, \bar{v}$ as desired, or the weakened formula is some $c_{i}^{\perp}$ and by induction hypothesis $\mathcal{A}, Q_{\ell} \triangleright_{\ell}\left(q, \bar{v}-\overline{\mathrm{e}}_{i}\right)$ and a loss allows to derive $\mathcal{A}, Q_{\ell} \triangleright_{\ell} q, \bar{v}$ as desired.

4.2.4. Contractive Case. Again, Fact 4.4 is straightforward to adapt to LLC+T using cut elimination. Fact 4.5 can be strengthened to avoid exponentials in the contractive case:

Lemma 4.8. For a finite set of axioms $T, \vdash \Gamma$ is provable in $L L C+T$ if and only if $\vdash \perp \oplus \bigoplus_{t \in T}\ulcorner t\urcorner, \Gamma$ is provable in $L L C$.

Proof. For the direct implication, we consider a directed proof of $\vdash_{\mathrm{LLC}+T} \Gamma$. By induction on the number of directed cuts, we build an LLC proof of $\vdash_{\text {LLC }} \perp \oplus \bigoplus_{t \in T}\ulcorner t\urcorner, \Gamma$. For the base case, an LLC $+T$ proof of $\vdash_{\mathrm{LLC}+T} \Gamma$ without directed cuts is also an LLC proof of $\vdash_{L L C} \Gamma$, thus $\vdash_{L L C} \perp, \Gamma$ using the $(\perp)$ rule, and $\vdash \perp \oplus \bigoplus_{t \in T}\ulcorner t\urcorner, \Gamma$ by $|T|$ applications of $(\oplus)$. For the induction step, consider a directed cut of an axiom $t=C, p_{1}^{\perp}, \ldots, p_{m}^{\perp}$ in $T$ with $\vdash_{\mathrm{LLC}+T} C^{\perp}, \Delta$. We have $\vdash_{\mathrm{LLC}} C, C^{\perp}$ and $\vdash_{\mathrm{LLC}} p_{i}, p_{i}^{\perp}$ for all $0<i \leq$ $m$ by the (init) rule, and $m+1$ applications of $(\otimes)$ yield $\vdash_{\text {LLC }} t,\ulcorner t\urcorner$. By induction hypothesis $\vdash_{\mathrm{LLC}} \perp \oplus \bigoplus_{t \in T}\ulcorner t\urcorner, C^{\perp}, \Delta$, thus a (normal) cut yields $\vdash_{\text {LLC }} \perp \oplus \bigoplus_{t \in T}\ulcorner t\urcorner,\ulcorner t\urcorner, p_{1}^{\perp}, \ldots, p_{m}^{\perp}, \Delta$. Using $|T|$ applications of ( $\left.\oplus\right)$ allows to prove $\vdash_{\text {LLC }} \perp \oplus \bigoplus_{t \in T}\ulcorner t\urcorner, \perp \oplus \bigoplus_{t \in T}\ulcorner t\urcorner, p_{1}^{\perp}, \ldots, p_{m}^{\perp}, \Delta$ and a structural contraction yields the desired LLC proof. 
For the converse implication, if $\vdash_{\mathrm{LLC}} \perp \oplus \bigoplus_{t \in T}\ulcorner t\urcorner, \Gamma$, then $\vdash_{\mathrm{LLC}+T} \perp \oplus$ $\bigoplus_{t \in T}\ulcorner t\urcorner, \Gamma$. Then $\vdash_{\text {LLC }+T} \mathbf{1}$, and for each axiom $t=C, p_{1}^{\perp}, \ldots, p_{m}^{\perp}$ in $T$, we can prove $\vdash_{\mathrm{LLC}+T} C \not \gamma p_{1}^{\perp} \not \gamma \ldots \gamma p_{m}^{\perp}$ by $m$ applications of $(\not)$ from $\vdash_{\mathrm{LLC}+T} t$, i.e. $\vdash_{\mathrm{LLC}+T}\ulcorner t\urcorner \perp$. Thus $|T|$ applications of (\&) yield $\vdash_{\mathrm{LLC}+T} \mathbf{1} \& \&_{t \in T}\ulcorner t\urcorner^{\perp}$, and a (normal) cut shows $\vdash_{\mathrm{LLC}+T} \Gamma$.

Without loss of generality, we can assume that $Q_{\ell}=\left\{q_{\ell}\right\}$ for a state $q_{\ell}$ with no applicable rule in $\mathcal{A}$. We extend Claim 4.6.1 and Proposition 4.6 to the contractive case:

Claim 4.9.1. For all $(q, \bar{v})$ in $Q \times \mathbb{N}^{d}, \mathcal{A},\left\{q_{\ell}\right\} \triangleright_{e} q, \bar{v}$ using expansive semantics if and only if $\vdash \theta(q, \bar{v}), q_{\ell}^{s}$ in LLC $+T$ for some $s>0$.

Proof. By Claim 4.6.1, it suffices to consider the case of expansions and structural contractions in a proof by induction over deduction tree height and number of directed cuts. In case of an expansion allowing to derive $\mathcal{A},\left\{q_{\ell}\right\} \triangleright_{e} q, \overline{\mathrm{v}}+\overline{\mathrm{e}}_{i}$, by induction hypothesis, $\vdash \theta\left(q, \overline{\mathrm{v}}+2 \overline{\mathrm{e}}_{i}\right), q_{\ell}^{s}$ and a structural contraction allows to prove $\vdash \theta\left(q, \overline{\mathrm{v}}+\overline{\mathrm{e}}_{i}\right), q_{\ell}^{s}$ as desired. Conversely, in case of a structural contraction proving $\vdash \theta(q, \bar{v}), q_{\ell}^{s}$, several cases are possible. If the contracted formula is $q_{\ell}$, then by induction hypothesis $\mathcal{A},\left\{q_{\ell}\right\} \triangleright_{e} q, \bar{v}$ as desired. If the contracted formula is some $e_{i}^{\perp}$ with $0<i \leq d$, then by induction hypothesis $\mathcal{A},\left\{q_{\ell}\right\} \triangleright_{e} q, \overline{\mathrm{v}}+2 \overline{\mathrm{e}}_{i}$ and an expansion allows to deduce $\mathcal{A},\left\{q_{\ell}\right\} \triangleright_{e} q, \overline{\mathrm{v}}+\overline{\mathrm{e}}_{i}$ as desired. Last of all, the contracted formula cannot be $q^{\perp}$ : assume for the sake of contradiction that $\vdash q_{1}^{\perp}, \ldots, q_{n}^{\perp}, \theta(q, \bar{v}), q_{\ell}$ were provable in LLC $+T$ for some $n>0$ negated atomic state propositions $q_{1}^{\perp}, \ldots, q_{n}^{\perp}$ (in addition to $q^{\perp}$ ), and attempt to perform directed proof search. The only applicable rules are

- structural contraction, which cannot decrease $n$, and

- directed cuts using $T$, which also preserve $n$.

In the absence of any axiom allowing $n>0$, this sequent is not provable.

Proposition 4.9. There is a logarithmic space reduction from $A B V A S S_{\overline{0}}$ expansive reachability to MALLC provability.

Proof. As usual, we start by eliminating full zero tests using Lemma 3.3. Let $\left\langle\mathcal{A}, q_{r},\left\{q_{\ell}\right\}\right\rangle$ be an expansive reachability instance. By Claim 4.9.1 and Lemma $4.8 \mathcal{A},\left\{q_{\ell}\right\} \triangleright_{e} q_{r}, \overline{0}$ if and only if $\vdash \perp \oplus \bigoplus_{t \in T}\ulcorner t\urcorner, q_{r}^{\perp}, q_{\ell}^{s}$ for some $s>0$, which by structural contractions on $q_{\ell}$ happens if and only if $\vdash$ $\perp \oplus \bigoplus_{t \in T}\ulcorner t\urcorner, q_{r}^{\perp}, q_{\ell}$.

\section{TOWER Upper Bounds}

To show that the reachability problem for lossy ABVASS $_{\overline{0}}$ is in Tower, we establish by induction over the dimension $d$ a bound on the height of minimal reachability witnesses, following in this the reasoning used by Rackoff [23] to show that the coverability problem for VASS is in ExPSPACE. The main new idea here is that, where there is freedom to choose how values of vector components are distributed when performing split rules top-down (see Section 3.1), splitting them equally (or with the difference of 1) allows sufficient lower bounds to be established along vertical paths in deduction 
trees for the inductive argument to go through. Since the bounds we obtain on the heights of smallest witnessing deduction trees are exponentiated at every inductive step (rather than multiplied as in Rackoff's proof), the resulting complexity upper bound involves a tower of exponentials, but will be shown broadly optimal in Section 6 .

The following lemma in fact addresses the equivalent top-down coverability problem (see $\S 3.2 .2$ ), and considers systems without full resets thanks to Lemma 3.3. We first define some terminology. We say that a deduction tree is:

- $\left(q_{r}, \overline{\mathrm{v}}_{0}\right)$-rooted iff that is the label of its root;

- $Q_{\ell}$-leaf-covering iff, for every leaf label $(q, \bar{v})$, we have $q \in Q_{\ell}$;

- of height $h$ iff that is the maximum number of edges, i.e. the maximum number of rule applications, along any path from the root to a leaf.

For integers $d, m \geq 0$ and $s \geq 1$, we define a natural number $H(d, s, m)$ recursively:

$$
\begin{aligned}
H(0, s, m) & \stackrel{\text { def }}{=} s \\
H(d+1, s, m) & \stackrel{\text { def }}{=} s\left(m \cdot 2^{H(d, s, m)}\right)^{d+1}+H(d, s, m) .
\end{aligned}
$$

Lemma 5.1. If an $A B V A S S \mathcal{A}=\left\langle Q, d, T_{u}, T_{f}, T_{s}, \emptyset\right\rangle$ has a $\left(q_{r}, \bar{v}_{0}\right)$-rooted $Q_{\ell}$-leaf-covering deduction tree, then it has such a deduction tree of height at most $H\left(d,|Q|, \max ^{-}\left(T_{u}\right)\right)$.

Proof. We use induction on the dimension $d$.

For the base case, if $\mathcal{A}$ is 0 -dimensional, then the labels in its deduction trees are states only. Starting with a deduction tree whose root label is $q_{r}$ and whose every leaf label is in $Q_{\ell}$, we obtain by repeated shortenings a deduction tree in which labels along every branch are mutually distinct, with height at most $|Q|-1$.

For the induction step in dimension $d+1$, suppose that $\mathcal{A}=\langle Q, d+$ $\left.1, T_{u}, T_{f}, T_{s}, \emptyset\right\rangle$, and $\mathcal{D}$ is a $\left(q_{r}, \bar{v}_{0}\right)$-rooted $Q_{\ell}$-leaf-covering deduction tree. Let

$$
B \stackrel{\text { def }}{=} 2^{H\left(d,|Q|, \max ^{-}\left(T_{u}\right)\right)} \cdot \max ^{-}\left(T_{u}\right),
$$

and let $\left\{n_{1}, \ldots, n_{k}\right\}$ be the set of all nodes of $\mathcal{D}$ such that, for all $i$, we have:

- all vector components in labels of ancestors of $n_{i}$ are smaller than $B$;

- for some $0<j_{i} \leq d+1$, we have $\overline{\mathbf{v}}_{i}\left(j_{i}\right) \geq B$, where the label of $n_{i}$ is $\left(q_{i}, \bar{v}_{i}\right)$.

By repeated shortenings, we can assume that the length (i.e., the number of edges) of every path in $\mathcal{D}$, which is from the root either to some $n_{i}$ or to a leaf with no $n_{i}$ ancestor, is at most $|Q| \cdot B^{d+1}$, the number of possible labels with all vector components smaller than $B$.

In the remainder of the argument, we apply the induction hypothesis below each of the nodes $n_{i}$. More precisely, let $\mathcal{A}_{i}$ denote the $d$-dimensional ABVASS obtained from $\mathcal{A}$ by projecting onto vector indices $\{1, \ldots, d+1\} \backslash$ $\left\{j_{i}\right\}$. (The only change is in the set of unary rules.) From the subtree of $\mathcal{D}$ rooted at $n_{i}$, we know that $\mathcal{A}_{i}$ has a $\left(q_{i}, \overline{\mathrm{v}}_{i}\left(-j_{i}\right)\right)$-rooted $Q_{\ell}$-leaf-covering 


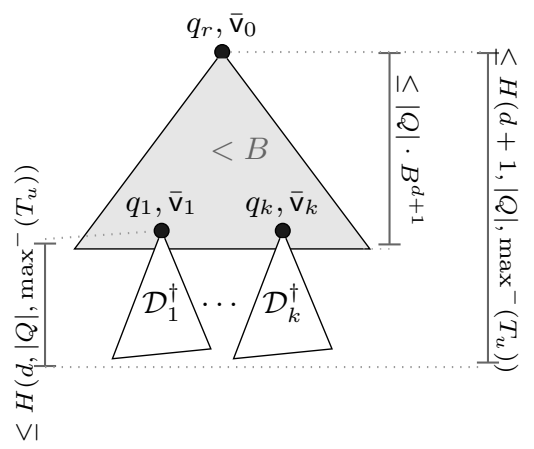

FiguRE 4. Induction step in the proof of Lemma 5.1.

deduction tree. (Here $\bar{w}(-j)$ denotes the projection of $\bar{w}$ to all indices except $j$.) Let $\mathcal{D}_{i}$ be such a deduction tree, which we can choose of height at most $H\left(d,|Q|, \max ^{-}\left(T_{u}\right)\right)$ by induction hypothesis.

Now, to turn $\mathcal{D}_{i}$ into a $\left(q_{i}, \overline{\mathrm{v}}_{i}\right)$-rooted deduction tree $\mathcal{D}_{i}^{\dagger}$ of $\mathcal{A}$, we have to do two things:

(1) For every application of a unary rule $q \stackrel{\bar{u}}{\rightarrow} q^{\prime}$ in $\mathcal{D}_{i}$, decide which unary rule $q \stackrel{\overline{\mathrm{u}}^{\prime}}{\rightarrow} q^{\prime}$ of $\mathcal{A}$ such that $\overline{\mathrm{u}}=\overline{\mathrm{u}}^{\prime}\left(-j_{i}\right)$ to apply: we do that arbitrarily.

(2) For every application of a split rule $q \rightarrow q^{\prime}+q^{\prime \prime}$ in $\mathcal{D}_{i}$, decide how to split the vector component $x$ with index $j_{i}$ : we do that by balancing, i.e. picking the corresponding components $x_{1}$ and $x_{2}$ of the two child vectors so that $\left|x_{1}-x_{2}\right| \leq 1$.

We claim that $\mathcal{D}_{i}^{\dagger}$ thus obtained is indeed a $\left(q_{i}, \overline{\mathrm{v}}_{i}\right)$-rooted $Q_{\ell^{-} \text {-leaf-covering }}$ deduction tree of $\mathcal{A}$. Since the node labels in $\mathcal{D}_{i}^{\dagger}$ differ from those in $\mathcal{D}_{i}$ only by the extra $j_{i}$ th components, it suffices to show that all the latter are non-negative. In fact, at the root of $\mathcal{D}_{i}^{\dagger}$, we have $\overline{\mathbf{v}}_{i}\left(j_{i}\right) \geq B$, and it follows by a straightforward induction that, for every node $n$ in $\mathcal{D}_{i}^{\dagger}$ whose distance from the root is $h$ (which is at most $H\left(d,|Q|, \max ^{-}\left(T_{u}\right)\right)$ ), its vector label $\overline{\mathrm{w}}$ satisfies

$$
\overline{\mathbf{w}}\left(j_{i}\right) \geq 2^{H\left(d,|Q|, \max ^{-}\left(T_{u}\right)\right)-h} \cdot \max ^{-}\left(T_{u}\right) .
$$

It remains to observe that, by replacing for each $0<i \leq k$, the subtree of $\mathcal{D}$ rooted at $n_{i}$ by $\mathcal{D}_{i}^{\dagger}$, the height of the resulting deduction tree (see Figure 4 for a depiction) is at most

$$
|Q| \cdot B^{d+1}+H\left(d,|Q|, \max ^{-}\left(T_{u}\right)\right)=H\left(d+1,|Q|, \max ^{-}\left(T_{u}\right)\right),
$$

thereby establishing the lemma.

The following auxiliary function and proposition will be useful for deriving the complexity upper bounds. Let

$$
H^{\prime}(d, s, m) \stackrel{\text { def }}{=} 4(d+1)(s+m+1) H(d, s, m) .
$$

Proposition 5.2. For all $d, m \geq 0$ and $s \geq 1$, we have:

$$
H^{\prime}(d+1, s, m) \leq 2^{H^{\prime}(d, s, m)} .
$$


Proof. We first observe the following inequality involving the $H$ function:

$$
\begin{aligned}
H(d+1, s, m) & =s\left(m \cdot 2^{H(d, s, m)}\right)^{d+1}+H(d, s, m) \\
& \leq s\left((m+1) \cdot 2^{H(d, s, m)}\right)^{d+1} \\
& \leq 2^{(d+1)(s+m+H(d, s, m))} \\
& \leq 2^{(d+1)(s+m+1) H(d, s, m)}
\end{aligned}
$$

and then use it to conclude that:

$$
\begin{aligned}
H^{\prime}(d+1, s, m) & =4(d+2)(s+m+1) H(d+1, s, m) \\
& \leq 4(d+2)(s+m+1) 2^{(d+1)(s+m+1) H(d, s, m)} \\
& \leq 2^{d+2} \cdot 2^{s+m+1} \cdot 2^{(d+1)(s+m+1) H(d, s, m)} \\
& \leq 2^{2(d+1)(s+m+1)} \cdot 2^{(d+1)(s+m+1) H(d, s, m)} \\
& \leq 2^{4(d+1)(s+m+1) H(d, s, m)} \\
& =2^{H^{\prime}(d, s, m)} .
\end{aligned}
$$

We are now in a position to establish the membership in Tower. More precisely, since the height of the tower of exponentials in the bounds we obtained is equal to the system dimension, the problem in fixed dimension $d$ is in $d$-ExpTIME.

Theorem 5.3. Reachability for lossy $A B V A S S_{\overline{0}}$ is in Tower. For every fixed dimension $d$, it is in PTIME if $d=0$, and in $d$-EXPTIME if $d \geq 1$.

Proof. By Lemma 3.3, it suffices to consider an ABVASS. We argue in terms of the top-down coverability problem (see $\S 3.2 .2$ ): given an ABVASS $\mathcal{A}=$ $\left\langle Q, d, T_{u}, T_{f}, T_{s}, \emptyset\right\rangle$, a state $q_{r}$ and a set of states $Q_{\ell}$, to decide whether $\mathcal{A}$ has a $\left(q_{r}, \overline{0}\right)$-rooted $Q_{\ell}$-leaf-covering deduction tree.

By Lemma 5.1, if $\mathcal{A}$ has such a deduction tree, then it has one of height at most $H\left(d,|Q|, \max ^{-}\left(T_{u}\right)\right)$. Observing that, in such a deduction tree, all vector components are bounded by

$$
\left(\max ^{+}\left(T_{u}\right)+1\right) \cdot H\left(d,|Q|, \max ^{-}\left(T_{u}\right)\right),
$$

we conclude that it can be guessed and checked in

$$
O\left((d+1) \cdot \log \left(\left(\max ^{+}\left(T_{u}\right)+1\right) \cdot H^{\prime}\left(d,|Q|, \max ^{-}\left(T_{u}\right)\right)\right)\right)
$$

space by an alternating algorithm which manipulates at most three configurations of $\mathcal{A}$ at a time.

The memberships in the statement (for ABVASS) follow from the fact that $H^{\prime}\left(0,|Q|, \max ^{-}\left(T_{u}\right)\right)$ is polynomial, by Proposition 5.2, and since ALoGSPACE $=$ PTime, APSpaCe $=$ ExpTime, and $(d-1)$-AExpSpaCe $=d$-ExPTime (see Chandra et al. [6]).

By Proposition 4.1, this shows:

Corollary 5.4. LLW provability is in TOWER. 


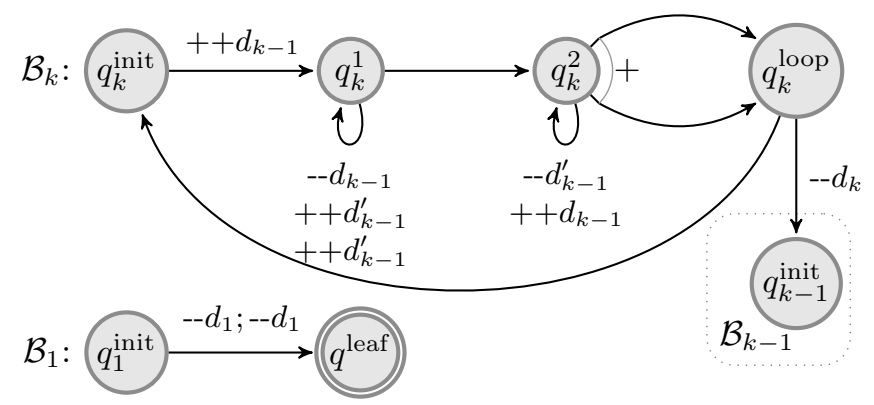

FiguRE 5. Defining $\mathcal{B}_{k}$ for $k>1$ (above), and $\mathcal{B}_{1}$ (below).

\section{TOWER LOWER Bounds}

The rough pattern of our hardness proof resembles those by e.g. Urquhart [30], Schnoebelen [28], where a fast-growing function is computed weakly, then its result is used to allocate space for simulating a universal machine, and finally the inverse of the function is computed weakly for checking purposes. Indeed, we simulate Minsky machines whose counters are towerbounded, but the novelty here is in the inverse computations. Specifically, for each Minsky counter $c$, we maintain its dual $\hat{c}$ and simulate each zero test on $c$ by a split rule that launches a thread to check that $\hat{c}$ has the maximum value. Recalling that such rules split all values non-deterministically, we must construct the simulating system carefully so that such non-determinism cannot result in erroneous behaviours.

The auxiliary threads check that a counter is at least tower $(k)$ by seeking to apply split rules at least tower $(k-1)$ times along every branch. The difficulty here is, similarly, how to count up to tower $(k-1)$ or more in a manner which is robust with respect to the non-determinism of the split rules.

A hierarchy of BVASS for the latter purpose is given in Figure 5 - recall the depicting conventions in $\S 3.1 .3$. In this system, after the unary rule from $q_{k}^{\text {loop }}$ that decrements $d_{k}$, we have that $\mathcal{B}_{k}$ behaves like $\mathcal{B}_{k-1}$ from state $q_{k-1}^{\text {init }}$.

Lemma 6.1. For every $k \geq 1$ and vector of naturals $\overline{\mathrm{v}}_{0}$ such that $\overline{\mathrm{v}}_{0}\left(d_{i}\right)=$ $\overline{\mathrm{v}}_{0}\left(d_{i}^{\prime}\right)=0$ for all $i<k$, we have that $\mathcal{B}_{k}$ has a $\left(q_{k}^{\text {init }}, \overline{\mathrm{v}}_{0}\right)$-rooted $\left\{q^{\text {leaf }}\right\}$-leafcovering deduction tree if and only if $\overline{\mathrm{v}}_{0}\left(d_{k}\right) \geq$ tower $(k)$.

Proof. We proceed by induction on $k$, where the base case $k=1$ is immediate, so let us consider $k>1$ and $\overline{\mathrm{v}}_{0}$ such that $\overline{\mathrm{v}}_{0}\left(d_{i}\right)=\overline{\mathrm{v}}_{0}\left(d_{i}^{\prime}\right)=0$ for all $i<k$.

If $\bar{v}_{0}\left(d_{k}\right) \geq$ tower $(k)$, we observe that $\mathcal{B}_{k}$ can proceed from $\left(q_{k}^{\text {init }}, \overline{\mathbf{v}}_{0}\right)$ as follows:

- each loop at $q_{k}^{1}$ empties $d_{k-1}$, i.e. doubles $d_{k-1}$ and transfers it to $d_{k-1}^{\prime}$;

- each loop at $q_{k}^{2}$ empties $d_{k-1}^{\prime}$, i.e. transfers $d_{k-1}^{\prime}$ back to $d_{k-1}$;

- each split from $q_{k}^{2}$ divides $d_{k-1}$ into two equal values, and divides $d_{k}$ into two values that differ by at most 1 . 
In any deduction tree thus obtained, at every node which is the $h$ th node with state label $q_{k}^{\text {loop }}$ from the root, and whose vector label is $\overline{\mathrm{w}}$, we have:

$$
\overline{\mathrm{w}}\left(d_{k-1}\right)=h, \quad \overline{\mathrm{w}}\left(d_{k-1}^{\prime}\right)=0, \quad \overline{\mathrm{w}}\left(d_{k}\right) \geq 2^{\text {tower }(k-1)-h} .
$$

Hence, by returning control to $q_{k}^{\text {init }}$ as long as the value of $d_{k}$ is at least 2 , $\mathcal{B}_{k}$ can reach along every vertical path a node with state label $q_{k}^{\text {loop }}$ at which the values of $d_{k-1}$ and $d_{k}$ are respectively equal to tower $(k-1)$ and at least 1. To complete the deduction tree to be $\left\{q^{\text {leaf }}\right\}$-leaf-covering, from every such node we let $\mathcal{B}_{k}$ decrement $d_{k}$ and apply the induction hypothesis on $\mathcal{B}_{k-1}$.

The interesting direction remains, so suppose $\mathcal{D}$ is a $\left(q_{k}^{\text {init }}, \bar{v}_{0}\right)$-rooted $\left\{q^{\text {leaf }}\right\}$-leaf-covering deduction tree of $\mathcal{B}_{k}$. Since at every $q_{k}^{\text {loop }}$-labelled node in $\mathcal{D}$, the value of $d_{k}$ must be at least 1 , it suffices to establish the following claim and apply it for the maximum $h$ :

Claim 6.1.1. For each $0<h \leq$ tower $(k-1)$, $\mathcal{D}$ contains $2^{h}$ incomparable nodes (i.e., none is a descendant of another) whose state label is $q_{k}^{\text {loop }}$ and at which $d_{k-1}+d_{k-1}^{\prime}$ has value at most $h$.

In turn, by induction on $h$, that claim is a straightforward consequence of the next one. (For the base case of that induction, i.e. $h=1$, apply the next claim with $h^{\prime}=0$.)

Claim 6.1.2. For each node $n$ in $\mathcal{D}$ whose state label is $q_{k}^{\text {init }}$ and at which $d_{k-1}+d_{k-1}^{\prime}$ has some value $h^{\prime}<$ tower $(k-1)$, there must be two incomparable descendants $n_{1}$ and $n_{2}$ whose state labels are $q_{k}^{\text {loop }}$ and at which the values of $d_{k-1}+d_{k-1}^{\prime}$ are at most $h^{\prime}+1$.

Consider a node $n$ as in the latter claim. After the increment of $d_{k-1}$ and the loops at $q_{k}^{1}$ and $q_{k}^{2}$, the value of $d_{k-1}+d_{k-1}^{\prime}$ will be at most $2\left(h^{\prime}+1\right)$. If the first split divides $d_{k-1}+d_{k-1}^{\prime}$ equally, we are done.

Otherwise, we have a $q_{k}^{\text {loop }}$-labelled descendant $n^{\prime}$ of $n$ at which $d_{k-1}+d_{k-1}^{\prime}$ has value at most $h^{\prime}$. In particular, $d_{k-1}$ is less than tower $(k-1)$ at $n^{\prime}$, so recalling the induction hypothesis regarding $\mathcal{B}_{k-1}$, the child $n^{\prime \prime}$ of $n^{\prime}$

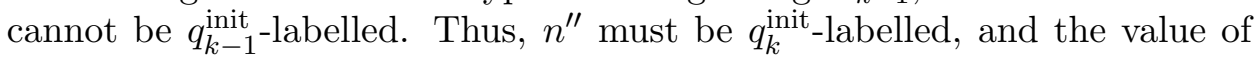
$d_{k-1}+d_{k-1}^{\prime}$ at $n^{\prime \prime}$ is the same as at $n^{\prime}$, so at most $h^{\prime}$. We can therefore repeat the argument with $n^{\prime \prime}$ instead of $n$, but since $\mathcal{D}$ is finite, two incomparable descendants as required eventually exist.

Relying on the properties of the BVASS $\mathcal{B}_{k}$, we now establish the hardness of lossy reachability, matching the membership in Tower in Theorem 5.3 already for BVASS. Although we do not match the upper bounds when the system dimension is fixed, we remark that our simulation uses a number of counters which is linear in the height of the tower of exponentials with coefficient 2 .

\section{Theorem 6.2. Reachability for lossy BVASS is ToweR-hard.}

Proof. For a notion of Minsky machines that is similar to how ABVASS $_{\overline{0}}$ were defined in Section 3.1, let such a machine be given by a finite set of states $Q$, a finite set of counters $C$, and finite sets of increment rules 


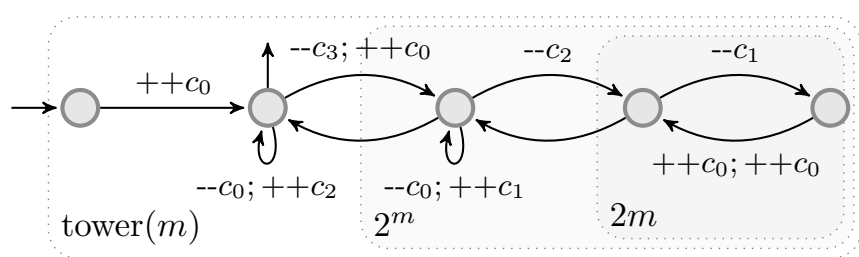

FIgURE 6. A VASS weakly computing tower $(m)$, with input counter $c_{3}$ and output counter $c_{0}$ [21]. Counter $c_{3}$ is initialised to $m$, and the others to 0 .

" $q \stackrel{++c}{\longrightarrow} q_{1}$, " decrement rules " $q \stackrel{--c}{\longrightarrow} q_{1}$ " and zero-test rules " $q \stackrel{c \stackrel{?}{=} 0}{\longrightarrow} q_{1}$. By simulating a tape using two stacks, and simulating a stack using two counters, it is straightforward to verify that the following problem [called $\mathbf{F}_{3}$-MM in 26] is Tower-hard:

Given a Minsky machine $\mathcal{M}$ and two states $q_{0}, q_{H}$, does $\mathcal{M}$ have a computation that starts in $q_{0}$ with all counters having value 0 , ends in $q_{H}$, and is such that all counter values are at most tower $(|\mathcal{M}|)$ ?

We establish the theorem by working with the equivalent top-down coverability problem (see $\$ 3.2 .2$ ). We show that, given a Minsky machine $\mathcal{M}$ of size $K$ and two states $q_{0}, q_{H}$, then a BVASS $\mathcal{A}(\mathcal{M})$, a state $q_{r}$ and a finite set $Q_{\ell} \stackrel{\text { def }}{=}\left\{q_{H}, q^{\text {leaf }}\right\}$ are computable in logarithmic space, such that $\mathcal{M}$ has a 0 -initialised tower $(K)$-bounded computation from $q_{0}$ to $q_{H}$ if and only if $\mathcal{A}(\mathcal{M})$ has a $\left(q_{r}, \overline{0}\right)$-rooted $Q_{\ell}$-leaf-covering deduction tree.

For each counter $c$ of $\mathcal{M}$, there are three counters in $\mathcal{A}(\mathcal{M})$ denoted $c, \hat{c}, c^{\prime}$. The initial part of $\mathcal{A}(\mathcal{M})$ employs a "weak Petri computer" [21] for the tower function, namely a constant VASS with designated input and output counters and initial and final states. Given a natural number $m$ in its input counter and starting in its initial state, it can compute tower $(m)$ in its output counter upon reaching its final state, but non-deterministically may also compute a smaller value (but never a larger one). This is a standard construction, using weak routines for $2 m$ and $2^{m}$, which we depict in Figure $6{ }^{3}$ By means of the latter VASS, each counter $\hat{c}$ in $\mathcal{A}(\mathcal{M})$ is initialised to have value tower $(K)$ or possibly smaller. Recalling that the auxiliary VASS is constant, a simple pattern for incorporating it into $\mathcal{A}(\mathcal{M})$ is to use fresh states and counters for each $\hat{c}$.

The main part of $\mathcal{A}(\mathcal{M})$ consists of simulating $\mathcal{M}$ from $q_{0}$, using the translations of increments, decrements and zero tests in Figure 7. For the increments and decrements, $\mathcal{A}(\mathcal{M})$ also performs the opposite operation on the hatted counter, thereby keeping the sums $c+\hat{c}$ constant. For the zero tests, $\mathcal{A}(\mathcal{M})$ attempts by two loops and using the primed counter, to copy the hatted counter to $d_{K}$ and then employ $\mathcal{B}_{K}$ (see Figure 5 to verify that the latter is maximal (i.e., has value tower $(K)$ ). Thus, $\mathcal{A}(\mathcal{M})$ also has counters $d_{i}$ for $0<i \leq K$ and $d_{i}^{\prime}$ for $0<i<K$, and more precisely a variant

\footnotetext{
${ }^{3}$ The reader puzzled by the " $++c_{0}$ " increments in Figure 6 should observe that tower $(0)=2^{0}=1$ but $2 \cdot 0=0$.
} 


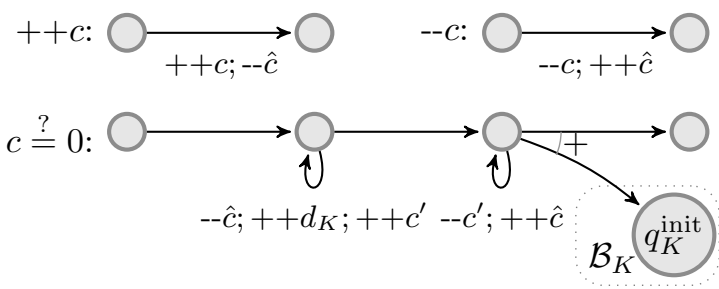

FiguRE 7. Simulating the Minsky operations.

of $\mathcal{B}_{K}$ is employed that has the same dimension as $\mathcal{A}(\mathcal{M})$ (and does not use the extra counters).

For each 0-initialised tower $(K)$-bounded computation of $\mathcal{M}$ from $q_{0}$ to $q_{H}$, it is straightforward to check that $\mathcal{A}(\mathcal{M})$ can simulate it as follows:

- each counter $\hat{c}$ is initialised to tower $(K)$;

- in every simulation of a zero test $c \stackrel{?}{=} 0$, the values of $c, \hat{c}, c^{\prime}, d_{K}$ are resp. 0 , tower $(K), 0,0$ before the two loops, and 0 , tower $(K), 0$, tower $(K)$ before the split;

- at every start of $\mathcal{B}_{K}$, the value of $d_{K}$ is tower $(K)$ and all other counters have value 0 .

By Lemma 6.1, we obtain a $\left(q_{r}, \overline{0}\right)$-rooted $Q_{\ell}$-leaf-covering deduction tree of $\mathcal{A}(\overline{\mathcal{M}})$.

The other direction is more involved: we show that, if $\mathcal{A}(\mathcal{M})$ has a $\left(q_{r}, \overline{0}\right)$-rooted $Q_{\ell}$-leaf-covering deduction tree $\mathcal{D}$, then $\mathcal{M}$ has a 0 -initialised tower $(K)$-bounded computation from $q_{0}$ to $q_{H}$. By construction, $\mathcal{D}$ consists of a path $\pi$ from which there are branchings to deduction trees of $\mathcal{B}_{K}$. The main part of $\pi$ consists of the simulations of increments, decrements and zero tests as in Figure 7 . From it, we obtain a 0 -initialised tower $(K)$-bounded computation of $\mathcal{M}$ from $q_{0}$ to $q_{H}$, after observing the following for every counter $c$ of $\mathcal{M}$ :

- After $\hat{c}$ is initialised in $\mathcal{D}$, the value of $c+\hat{c}+c^{\prime}$ is always at most tower $(K)$.

- For each simulation of a zero test of $c$, we have by Lemma 6.1 that the value of $d_{K}$ is tower $(K)$ before the split and is 0 after the split on the path $\pi$, and consequently that the values of $c, \hat{c}, c^{\prime}$ are 0 , tower $(K), 0$ (respectively) before the two loops.

- The value of $c$ may erroneously decrease due to the branchings, but since that makes the value of $c+\hat{c}+c^{\prime}$ smaller than tower $(K)$, such losses may occur only after the last simulation of a zero test of $c$, and so cannot result in an erroneous such simulation.

- Similarly, only the last transfer of $c^{\prime}$ to $\hat{c}$ may be incomplete (i.e., it does not empty $c^{\prime}$ ).

We conclude that $\mathcal{A}(\mathcal{M})$ has the required properties.

Since lossy reachability reduces to reachability and by Proposition 4.6 and Proposition 4.7, this entails:

Corollary 6.3. Provability in MELL, MELLW, and $L L W$ is Tower-hard. 


\section{Ackermann Upper Bounds}

We investigate in this section the complexity of reachability in increasing or expansive ABVASS $_{\overline{0}}$. The latter is related to provability in contractive linear logic, and as shown by Urquhart [30], to provability in the conjunctiveimplicative fragment of relevance logic, and we treat it in Section 7.3.

7.1. Increasing Reachability. Expansive $\operatorname{ABVASS}_{\overline{0}}$ are not quite dual to lossy $\mathrm{ABVASS}_{\overline{0}}$ : the natural model for this is that of increasing $\mathrm{ABVASS}_{\overline{0}}$, which feature additional deduction rules

$$
\frac{q, \overline{\mathrm{v}}}{q, \overline{\mathrm{v}}+\overline{\mathrm{e}}_{i}} \text { increase }
$$

for all $q$ in $Q$ and $0<i \leq d$.

7.1.1. Bottom-Up Coverability. As with lossy reachability, increasing reachability corresponds to a coverability problem in a variant of $\mathrm{ABVASS}_{\overline{0}}$. Let us define a variant of ABVASS $_{\overline{0}}$ that provides different semantics

- for rules in $T_{f}$ as meets " $q \rightarrow q_{1} \sqcap q_{2}$ " instead of forks, and

- for rules in $T_{z}$ as zero-jumps " $q \stackrel{\bar{\omega}}{\rightarrow} q_{1}$ " instead of full zero tests.

Their semantics are now defined by the deduction rules

$$
\frac{q, \overline{\mathrm{v}}_{1} \sqcap \overline{\mathrm{v}}_{2}}{q_{1}, \overline{\mathrm{v}}_{1} \quad q_{2}, \overline{\mathrm{v}}_{2}} \text { meet } \quad \frac{q, \overline{0}}{q_{1}, \overline{\mathrm{v}}} \text { zero-jump }
$$

where the meet $\overline{\mathrm{v}}_{1} \sqcap \overline{\mathrm{v}}_{2}$ of two vectors in $\mathbb{N}^{d}$ is the component-wise minimum of $\overline{\mathrm{v}}_{1}$ and $\overline{\mathrm{v}}_{2}$ : for all $0<i \leq d$,

$$
\left(\overline{\mathrm{v}}_{1} \sqcap \overline{\mathrm{v}}_{2}\right)(i) \stackrel{\text { def }}{=} \min \left(\overline{\mathrm{v}}_{1}(i), \overline{\mathrm{v}}_{2}(i)\right) .
$$

Let us call the resulting model ABVASSi. Given an ABVASSi $\mathcal{A}$, a state $q_{r}$, and a finite set of states $Q_{\ell}$, the bottom-up coverability or root coverability problem asks for the existence of a deduction tree $\mathcal{D}$ with root label $\left(q_{r}, \bar{v}\right)$ for some $\bar{v}$ in $\mathbb{N}^{d}$ where $d$ is the dimension of $\mathcal{A}$, and with every leaf labelled by some element of $Q_{\ell} \times\{\overline{0}\}$.

By a reasoning similar to the one employed for top-down coverability in ABVASSr, bottom-up coverability in ABVASSi corresponds to increasing reachability in ABVASS $_{\overline{0}}$ : by monotonicity we can always increase as soon as possible in the latter, either at the root, or right after a full zero test, or right after an "imbalanced" fork - where increases differ on the two branches.

7.1.2. Pseudo-Increasing $A B V A S S_{\overline{0}}$. Let us consider yet another variant of increasing ABVASS $_{\overline{0}}$, which will be used in the complexity analysis, and which combines increasing steps with unary steps. Given a vector $\overline{\mathrm{u}}$ in $\mathbb{Z}^{d}$, let us denote by $\overline{\mathrm{u}}_{-}$the vector in $\mathbb{N}^{d}$ defined for all $0<i \leq d$ by

$$
\overline{\mathrm{u}}_{-}(i) \stackrel{\text { def }}{=} \begin{cases}-\overline{\mathrm{u}}(i) & \text { if } \overline{\mathrm{u}}(i)<0 \\ 0 & \text { otherwise. }\end{cases}
$$

Then, for a vector $\overline{\mathrm{v}}$ in $\mathbb{N}^{d}$, the join $\overline{\mathrm{v}} \sqcup \overline{\mathrm{u}}_{-}$defined for all $0<i \leq d$ by

$$
\left(\overline{\mathrm{v}} \sqcup \overline{\mathrm{u}}_{-}\right)(i) \stackrel{\text { def }}{=} \max \left(\overline{\mathrm{v}}(i), \overline{\mathrm{u}}_{-}(i)\right)
$$

is the minimal vector greater or equal to $\bar{v}$ that allows to fire a unary rule with vector $\bar{u}$. 
A pseudo-increasing $\mathrm{ABVASS}_{\overline{0}}$ does not have the increasing rule, but uses instead a different semantics for its unary rules $q \stackrel{\bar{u}}{\rightarrow} q^{\prime}$ in $T_{u}$, which can be used for any $\overline{\mathrm{v}}$ in $\mathbb{N}^{d}$ :

$$
\frac{q, \overline{\mathrm{v}}}{q^{\prime},\left(\overline{\mathrm{v}} \sqcup \overline{\mathrm{u}}_{-}\right)+\overline{\mathrm{u}}} \text { pseudo-unary }
$$

The idea of the pseudo-unary rule is that it implicitly applies the minimal amount of increase necessary to use a given unary rule.

Reachability (from $\left(q_{r}, \overline{0}\right)$ to $Q_{\ell} \times\{\overline{0}\}$ ) in an increasing ABVASS $_{\overline{0}}$ is then equivalent to reachability in the same $\mathrm{ABVASS}_{\overline{0}}$ with pseudo-increasing semantics. We can indeed delay increases occurring right before another rule: an increase right before a fork or a split can be performed after it, no increase can occur right before a full zero test, and superfluous increases right before a unary rule $q \stackrel{\overline{\mathrm{u}}}{\rightarrow} q^{\prime}$ can be performed after it, i.e. an increase from $(q, \overline{\mathrm{v}})$ to $\left(q, \overline{\mathrm{v}}+\overline{\mathrm{e}}_{i}\right)$ with $\overline{\mathrm{v}} \geq \overline{\mathrm{u}}_{-}$can rather be performed from $\left(q^{\prime}, \overline{\mathrm{v}}+\overline{\mathrm{u}}\right)$ to $\left(q^{\prime}, \overline{\mathrm{v}}+\overline{\mathrm{e}}_{i}+\overline{\mathrm{u}}\right)$. The remaining increases right before unary rules become part of pseudo-unary rules.

7.2. Complexity of Increasing Reachability. In the more restricted case of BVASS, which do not feature forks nor full zero tests, bottom-up coverability coincides with increasing reachability, and this problem was called more simply "coverability" by Verma and Goubault-Larrecq [32]:

Fact 7.1 (Demri et al. [10]). Reachability in increasing BVASS is 2-ExpTimecomplete.

Since increasing $\mathrm{ABVASS}_{\overline{0}}$ are not too different from expanding $\mathrm{ABVASS}_{\overline{0}}$, the fact that their complexity is the same is not too surprising:

Theorem 7.2. Reachability in increasing AVASS and increasing $A B V A S S_{\overline{0}}$ is ACKeRMAnN-complete.

Proof. For the lower bound, a reduction from the reachability problem in increasing Minsky machines [28] is straightforward, since it uses the same encoding of zero tests as the proof sketch for Fact 3.2.

For the upper bound, by Lemma 3.3, we can restrict ourselves to ABVASS without loss of generality. Define the partial order $\leq$ over configurations in $Q \times \mathbb{N}^{d}$ by $(q, \overline{\mathrm{v}}) \leq\left(q^{\prime}, \overline{\mathrm{v}}^{\prime}\right)$ if $q=q^{\prime}$ and $\overline{\mathrm{v}} \leq \overline{\mathrm{v}}^{\prime}$; this is the product ordering over $Q \times \mathbb{N}^{d}$, where $Q$ is ordered using equality. By Dickson's Lemma, $\left(Q \times \mathbb{N}^{d}, \leq\right)$ is a well quasi order. The proof for the upper bound thus follows the typical steps of an application of the length function theorem for Dickson's Lemma found in [12].

Let us consider an increasing reachability witness $\mathcal{D}$ and the sequence of labels $\left(q_{r}, \overline{0}\right)=\left(q_{0}, \bar{v}_{0}\right),\left(q_{1}, \bar{v}_{1}\right), \ldots,\left(q_{m}, \bar{v}_{m}\right) \in Q_{\ell} \times\{\overline{0}\}$ along some branch $n_{0}, n_{1}, \ldots, n_{m}$ of $\mathcal{D}$. By ignoring the intermediate increase steps, we extract a pseudo-subsequence $\left(q_{i_{0}}, \overline{\mathrm{v}}_{i_{0}}\right),\left(q_{i_{1}}, \overline{\mathrm{v}}_{i_{1}}\right), \ldots,\left(q_{i_{p}}, \overline{\mathrm{v}}_{i_{p}}\right)$ with $\left(q_{i_{0}}, \overline{\mathrm{v}}_{i_{0}}\right) \geq\left(q_{r}, \overline{0}\right)$ and, for each $0<j \leq p,\left(q_{i_{j}}, \overline{\mathrm{v}}_{i_{j}}\right) \geq\left(q_{i_{j-1}+1}, \overline{\mathrm{v}}_{i_{j-1}+1}\right)$.

Assume now that $\mathcal{D}$, among all the increasing reachability witnesses, has pseudo-subsequences of minimal length (noted $p+1$ above) along each branch. Then, along any branch, for all $0 \leq j<k \leq p,\left(q_{i_{j}}, \bar{v}_{i_{j}}\right) \not \leq\left(q_{i_{k}}, \bar{v}_{i_{k}}\right)$, or a sequence of increases would allow to go from $n_{i_{j-1}}$ to $n_{i_{k}}$ directly with 
a strictly shorter pseudo-subsequence. In terms of the wqo, this means that, along any branch, the pseudo-subsequence is a bad sequence. Let us furthermore apply the strategy in $\$ 7.1 .2$ and delay increases as much as possiblenote that this might provide further opportunities for reducing the length of pseudo-sequences along the branches of $\mathcal{D}$. The resulting increasing reachability witness, where the remaining increases occur necessarily just before unary rules, can then be seen as a pseudo-increasing reachability witness, where every sequence of labels along every branch is a bad sequence for the wqo $\left(Q \times \mathbb{N}^{d}, \leq\right)$.

Define the norm $\|q, \bar{v}\|$ of a configuration $(q, \bar{v})$ in $Q \times \mathbb{N}^{d}$ as the infinite norm $\max _{0<i \leq d} \overline{\mathbf{v}}(i)$ of $\overline{\mathrm{v}}$. Observe that, along any branch of a pseudoincreasing reachability witness, if $\left(q_{j}, \bar{v}_{j}\right)$ and $\left(q_{j+1}, \overline{\mathrm{v}}_{j+1}\right)$ are two successive labels, then

$$
\left\|q_{j+1}, \overline{\mathrm{v}}_{j+1}\right\| \leq\left\|q_{j}, \overline{\mathrm{v}}_{j}\right\|+\max ^{-}\left(T_{u}\right)+\max ^{+}\left(T_{u}\right) .
$$

Define accordingly $g(x) \stackrel{\text { def }}{=} x+\max ^{-}\left(T_{u}\right)+\max ^{+}\left(T_{u}\right)$, then for the $j$ th label along a branch, $\left\|q_{j}, \bar{v}_{j}\right\| \leq g^{j}(0)$ the $j$ th iterate of $g$. This shows that the sequence of labels along every branch of our pseudo-increasing reachability witness is a bad sequence controlled by $(g, 0)$.

A length function theorem for a wqo is a combinatorial statement bounding the length of bad controlled sequences. In our case, for the wqo $\left(Q \times \mathbb{N}^{d}, \leq\right)$ and the control $(g, 0)$, the theorem in [12] yields an

$$
F_{d+1}\left(p\left(\max ^{-}\left(T_{u}\right)+\max ^{+}\left(T_{u}\right),|Q|\right)\right) \leq \operatorname{Ack}\left(p^{\prime}(|\mathcal{A}|)\right)
$$

upper bound on the length of branches for some polynomial functions $p$ and $p^{\prime}$, where $\left(F_{d}: \mathbb{N} \rightarrow \mathbb{N}\right)_{d}$ is a hierarchy of fast-growing functions [20] with $\operatorname{Ack}(n) \stackrel{\text { def }}{=} F_{n+1}(n)$. A non-deterministic combinatorial algorithm can thus compute the bound in (20) and attempt to find a pseudo-increasing witness of such bounded height (note that the branching degree of witnesses is also bounded) in Ackermannian time. As with lossy reachability, the main parameter in this complexity upper bound is the dimension $d$ of the $\operatorname{ABVASS}_{\overline{0}}$.

7.3. Complexity of Expansive Reachability. Turning to expansive reachability, we present now the missing proof of Theorem 3.7

Theorem 3.7. Reachability in expansive AVASS and expansive ABVASS$S_{\overline{0}}$ is ACKERMANN-complete.

Proof. The lower bound is proved by Urquhart [30]. The upper bound is also similar to that of Urquhart for provability in LR+, and follows essentially the same scheme as in the increasing case in the proof of Theorem 7.2. By Lemma 3.3 we restrict ourselves to ABVASS. Define the partial order $\sqsubseteq$ over configurations in $Q \times \mathbb{N}^{d}$ by $(q, \bar{v}) \sqsubseteq\left(q^{\prime}, \bar{v}^{\prime}\right)$ if $q=q^{\prime}, \bar{v} \leq \bar{v}^{\prime}$, and $\sigma(\overline{\mathrm{v}})=\sigma\left(\overline{\mathrm{v}}^{\prime}\right)$, where $\sigma(\overline{\mathrm{v}}) \stackrel{\text { def }}{=}\{0<i \leq d \mid \overline{\mathrm{v}}(i)>0\}$ denotes the support of $\overline{\mathrm{v}}$. The quasi-order $\left(Q \times \mathbb{N}^{d}, \sqsubseteq\right)$ is isomorphic to the sub-order of the product ordering over $Q \times \mathbb{N}^{d} \times 2^{d}$ induced by the restriction to triples $(q, \bar{v}, s)$ where $\sigma(\overline{\mathrm{v}})=s$, and is therefore a wqo by Dickson's Lemma.

Substituting $\sqsubseteq$ for $\leq$, we show as in the proof of Theorem 7.2 that, if there is an expansive reachability witness $\mathcal{D}$, then there is one where pseudo 
TABLE 1. The complexity of provability in fragments and variants of LL.

\begin{tabular}{lcc}
\hline & MELL & LL \\
\hline & Tower-hard, $\Sigma_{1}^{0}$-easy & $\Sigma_{1}^{0}$-c. [18] \\
with W & TowER-c. & TOwER-c. \\
with C & 2EXP-c. [27] & ACK-c. \\
\hline
\end{tabular}

TABLE 2. The complexity of reachability problems in $\operatorname{ABVASS}_{\overline{0}}$.

\begin{tabular}{lccc}
\hline & AVASS & BVASS & ABVASS $_{\overline{0}}$ \\
\hline Reachability & $\Sigma_{1}^{0}$-c. $[18]$ & Tower-hard, $\Sigma_{1}^{0}$-easy & $\Sigma_{1}^{0}$-c. \\
Lossy reach. & 2ExP-c. [8] & ToweR-c. & TowER-c. \\
Incr. reach. & ACK-c. [30] & 2ExP-c. [10] & ACK-c. \\
\hline
\end{tabular}

sequences are bad sequences for $\left(Q \times \mathbb{N}^{d}\right.$, $)$ along each branch, and where expansions are applied as late as possible. Thus $\mathcal{D}$ can be seen as a pseudoexpansive reachability witness, using the semantics of pseudo-unary rules for unary rules in $T_{u}$, with the additional restriction that such a rule can only be applied if $\sigma\left(\overline{\mathrm{u}}_{-}\right) \subseteq \sigma(\overline{\mathrm{v}})$. This restriction reflects the fact that expansions cannot increase a zero coordinate in $\bar{v}$. The remaining steps are the same as in the proof of Theorem 7.2 the sequences of labels along the branches of $\mathcal{D}$ are bad $(g, 0)$-controlled sequences for $\left(Q \times \mathbb{N}^{d}, \sqsubseteq\right)$, and we obtain similarly an

$$
F_{d+1}\left(p\left(\max ^{-}\left(T_{u}\right)+\max ^{+}\left(T_{u}\right),|Q| \cdot 2^{d}\right)\right) \leq \operatorname{Ack}\left(p^{\prime}(|\mathcal{A}|)\right)
$$

upper bound on the height of our witness, for some polynomial functions $p$ and $p^{\prime}$. Again, the main complexity parameter is the dimension $d$ of the ABVASS $_{\overline{0}}$.

Corollary 7.3. MALLC and LLC provability are ACKERMANN-complete.

Proof. By Theorem 3.7 and the reductions from LLC provability to ABVASS $_{\overline{0}}$ expansive reachability in Proposition 4.1 and from AVASS expansive reachability to MALLC provability in Proposition 4.9.

\section{Concluding Remarks}

Although connections between propositional linear logic and families of counter machines have long been known, they have rarely been exploited for complexity-theoretic results. Using a model of alternating branching VASS, we have unified several of these connections, and derived complexity bounds for provability in substructural logics from the (old and new) bounds on ABVASS $_{\overline{0}}$ reachability, summarised in Table 1 and Table 2 respectively.

Our main results in this regard are the TOWER-completeness of provability in LLW and the new TOwER lower bound for MELL: the latter has consequences on numerous problems mentioned in Section 3, and entails for instance that the satisfiability problem for $\mathrm{FO}^{2}$ on data trees is nonelementary [3, 11]. The ACKERMANN-completeness of MALLC and LLC is 
perhaps less surprising in the light of Urquhart's results, but we take it as a testimony to the versatility of the ABVASS $_{\overline{0}}$ model.

The main open question remains whether BVASS reachability, or equivalently MELL provability, is decidable.

\section{REFERENCES}

[1] P. A. Abdulla, R. Mayr, A. Sangnier, and J. Sproston. Solving parity games on integer vectors. In P. R. D'Argenio and H. Melgratti, editors, Concur 2013, volume 8052 of Lect. Notes in Comput. Sci., pages 106-120. Springer, 2013. doi:10.1007/ 978-3-642-40184-8_9.

[2] A. Asperti and L. Roversi. Intuitionistic light affine logic. ACM Trans. Comput. Logic, 3(1):137-175, 2002. doi:10.1145/504077.504081

[3] M. Bojańczyk, A. Muscholl, T. Schwentick, and L. Segoufin. Two-variable logic on data trees and XML reasoning. J. ACM, 56(3):1-48, 2009. doi:10.1145/ 1516512.1516515 .

[4] A. Bouajjani and M. Emmi. Analysis of recursively parallel programs. ACM Trans. Prog. Lang. Syst., 35(3):10:1-10:49, 2013. doi:10.1145/2518188.

[5] T. Brázdil, P. Jančar, and A. Kučera. Reachability games on extended vector addition systems with states. In S. Abramsky, C. Gavoille, C. Kirchner, F. Meyer auf der Heide, and P. G. Spirakis, editors, ICALP 2010, volume 6199 of Lect. Notes in Comput. Sci., pages 478-489. Springer, 2010. doi:10.1007/978-3-642-14162-1_40

[6] A. K. Chandra, D. C. Kozen, and L. J. Stockmeyer. Alternation. J. ACM, 28(1): 114-133, 1981. doi:10.1145/322234.322243

[7] K. Chatterjee, L. Doyen, T. A. Henzinger, and J.-F. Raskin. Generalized meanpayoff and energy games. In K. Lodaya and M. Mahajan, editors, FSTTCS 2010, volume 8 of Leibniz Int. Proc. Inf., pages 505-516. LZI, 2010. doi:10.4230/ LIPIcs.FSTTCS.2010.505.

[8] J.-B. Courtois and S. Schmitz. Alternating vector addition systems with states. In E. Csuhaj-Varjú, M. Dietzfelbinger, and Z. Ésik, editors, MFCS 2014, volume 8634 of Lect. Notes in Comput. Sci., pages 220-231. Springer, 2014. doi:10.1007/ 978-3-662-44522-8_19.

[9] Ph. de Groote, B. Guillaume, and S. Salvati. Vector addition tree automata. In LICS 2004, pages 64-73. IEEE Computer Society, 2004. ISBN 0-7695-2192-4. doi: 10.1109/LICS.2004.51.

[10] S. Demri, M. Jurdziński, O. Lachish, and R. Lazić. The covering and boundedness problems for branching vector addition systems. J. Comput. Syst. Sci., 79(1):23-38, 2013. doi:10.1016/j.jcss.2012.04.002.

[11] J. Dimino, F. Jacquemard, and L. Segoufin. $\mathrm{FO}^{2}(<,+1, \sim)$ on data trees, data tree automata and an extension of BVASS. Manuscript, 2013. URL http://hal.inria.fr/ hal-00769249.

[12] D. Figueira, S. Figueira, S. Schmitz, and Ph. Schnoebelen. Ackermannian and primitive-recursive bounds with Dickson's Lemma. In LICS 2011, pages 269-278. IEEE Computer Society, 2011. doi:10.1109/LICS.2011.39

[13] M. I. Kanovich. Petri nets, Horn programs, linear logic and vector games. Ann. Pure App. Logic, 75(1-2):107-135, 1995. doi:10.1016/0168-0072(94)00060-G.

[14] A. P. Kopylov. Decidability of linear affine logic. Inform. and Comput., 164(1): 173-198, 2001. doi:10.1006/inco.1999.2834

[15] Y. Lafont. The finite model property for various fragments of linear logic. J. Symb. Log., 62(4):1202-1208, 1997. doi:10.2307/2275637.

[16] D. Larchey-Wendling and D. Galmiche. Nondeterministic phase semantics and the undecidability of Boolean BI. ACM Trans. Comput. Logic, 14(1):6:1-6:41, 2013. doi:10.1145/2422085.2422091.

[17] R. Lazić. The reachability problem for branching vector addition systems requires doubly-exponential space. Inf. Process. Lett., 110(17):740-745, 2010. doi:10.1016/ j.ipl.2010.06.008 
[18] P. Lincoln, J. Mitchell, A. Scedrov, and N. Shankar. Decision problems for propositional linear logic. Ann. Pure App. Logic, 56(1-3):239-311, 1992. doi:10.1016/ 0168-0072(92)90075-B.

[19] R. Lipton. The reachability problem requires exponential space. Technical Report 62, Yale University, 1976.

[20] M. H. Löb and S. S. Wainer. Hierarchies of number theoretic functions, I. Arch. Math. Logic, 13:39-51, 1970. doi:10.1007/BF01967649

[21] E. W. Mayr and A. R. Meyer. The complexity of the finite containment problem for Petri nets. J. ACM, 28(3):561-576, 1981. doi:10.1145/322261.322271.

[22] M. Okada and K. Terui. The finite model property for various fragments of intuitionistic linear logic. J. Symb. Log., 64(2):790-802, 1999. doi:10.2307/2586501.

[23] C. Rackoff. The covering and boundedness problems for vector addition systems. Theor. Comput. Sci., 6(2):223-231, 1978. doi:10.1016/0304-3975(78)90036-1.

[24] O. Rambow. Multiset-valued linear index grammars: imposing dominance constraints on derivations. In ACL '94, pages 263-270. ACL Press, 1994. doi: $10.3115 / 981732.981768$

[25] S. Schmitz. On the computational complexity of dominance links in grammatical formalisms. In $A C L$ 2010, pages 514-524. ACL Press, 2010. URL http: //hal.archives-ouvertes.fr/hal-00482396.

[26] S. Schmitz. Complexity hierarchies beyond Elementary. ACM Trans. Comput. Theory, (1):1-36, 2016. doi:10.1145/2858784. URL http://arxiv.org/abs/1312.5686

[27] S. Schmitz. Implicational relevance logic is 2-ExPTIME-complete. J. Symb. Log., 81 (2):641-661, 2016. doi:10.1017/jsl.2015.7. URL http://arxiv.org/abs/1402.0705

[28] Ph. Schnoebelen. Revisiting Ackermann-hardness for lossy counter machines and reset Petri nets. In P. Hliněný and A. Kučera, editors, MFCS 2010, volume 6281 of Lect. Notes in Comput. Sci., pages 616-628. Springer, 2010. doi:10.1007/ 978-3-642-15155-2_54.

[29] A. S. Troelstra. Lectures on Linear Logic. CSLI, 1992.

[30] A. Urquhart. The complexity of decision procedures in relevance logic II. J. Symb. Log., 64(4):1774-1802, 1999. doi:10.2307/2586811.

[31] A. Urquhart. The complexity of linear logic with weakening. In S. Buss, P. Hajek, and P. Pudlak, editors, Logic Colloquium '98, volume 13 of Lect. Notes in Logic, pages 500-515. ASL, 2000.

[32] K. N. Verma and J. Goubault-Larrecq. Karp-Miller trees for a branching extension of VASS. Disc. Math. Theor. Comput. Sci., 7(1):217-230, 2005.

Department of Computer Science, University of WARWick, UK

Email address: lazic@dcs.warwick.ac.uk

LSV, ENS CACHAN \& CNRS \& INRIA, France

Email address: schmitz@lsv.ens-cachan.fr 